Zur Theorie der orthogonalen Substitutionen.

Von

A. Voss in Darmstadt.

Die Theorie der orthogonalen Substitutionen ist bereits vielfach behandelt und durch die berühmten Cayley'schen Formeln, vermöge deren sich die Coefficienten einer allgemeinen orthogonalen Substitution a priori angeben lassen, zu einem gewissen Abschluss gebracht. Es ist bekannt, in welch' enger Beziehung dieselbe zu der weiteren Aufgabe steht, eine quadratische Form in sich selbst zu transformiren (Hermite'sche Transformation), ein Problem, welebes nicht allein für drei und rier homogene $V$ ariabele eine anschauliche Bedeutung in der Ebene and im Raume, sondern auch namentlich für sechs eine geometrische Repräsentation in der Transformation des Linienranmes findet. Indem ich die Coefficienten der Gleichung einer durch ihre Tangenten dargestellten Fläche zweiten Grades als Coefficienten einer speciellen (symmetrischen) orthogronalen Substitution erkannte, ergab sich Veranlassung gewisse Determinanten solcher Coefficienten genauer 2n untersuchen.*) Ich habe seitdem bemerkt, dass eine ganze Reihe. der in einer früheren Arbeit ausgeführten Betrachtungen sich auf orthogonale Substitutionen überhanpt ausdehnen Jassen, deren Ausfürung den Gegenstand der vorliegenden Arbeit bildet.**)

In $\$$ I. sind die bekannten Bedingungen für die Coefficienten $a_{i k}$ einer orthogonalen Substitution aufgestellt, dorch welche eine Fläche 2weiten Grades in sich transformirt wird, und die Beziehungen zwischen den Elementen entwickelt, welche bei der Transformation fest bleiben. Daran schliesst sich in \$ II. eine allgemeine Untersuchang der Untesdeterminantensysteme soleher Substitutionen, welche, wie ich glaube, geeignet ist, die verschiedenen Fälle, die überhaupt bei ihnen auf-

- Math Annalen X.

*) Enst nach Vollendung derselben (Jnni 1877) erschien die umfangreiche neqeate Arbeit des Herra Frobenius: "Ueber lineare Substitutionen and bilineare Formen," Cralle $\$ 1,1$. 
treten können, a priori übersehen za lassen. Insbesondere erwälme ich eine dabei hervortretende directe Ableitung der Caylay'schen Formeln selbst.

In dem folgenden $\$$ III. sind die quadratïsehen formen, weldhe gleichzeitig durch die Substitution in sich transformirt werden, hesprochen, sowie die Gruppe der mit ciner geghbeves Substitudion pertauschbaren allgemein hergeleitet. Inshesondere habe ich dabei versudht der merkwürdigen Zerlegung der Trausformation einer Flache zweiten Grades im gewöbnlichen Raume, nach welchẹr man eine jede aus zwei speciellen vertauschbaren Transformationen zusammensetzen kanu, bei denen je ein System vou Erzeugenden fest bleibt, ein Analogon in böheren Räumen zu geben. $\$ V$. untersucht Transformationen von speciellem, Charalter, nameatlich die wneigoulicht', welche als Combal. projection der Fläche in sich selbst ron einum beliebigen Puldte aus aufgefasst werden kann, und die symptrische, bei welcher die Coeffi. cienten $a_{i k}$ und $a_{k i}$ gleich sind. Weiter ist das Problem der zorlegkng der Sulstitutionen behondelt, namentlich gezeigt, wie jods allgomeno durch eine gewisse Zahl ureigentlicher speciellen, d. h. durch wiederholte Centralprojectionen von Punkten ans, die nach einer selpreinfachen Regel gefunden werden können, oder durch stoe symmetrische ersetzt werden können, welch' letztere wieder atus einer Gruppe wertauschbarer Centralprojectionen hervorgehen.

In $\$$ VI. sind die besonderen falle der Substitutionen bei 2,8 , 4, 6 homogenen Variabeln behandelt und geometrisch interpretirt; zugleieh habe ich versucht sämmtliche Substitationen dieser Art aufzuzählen. Dabei ergeben sich gewisse Eigenschaften der reciproken Punkt-Ebenentransformation, welche, obwoh! in naher Beziehung za von den Herren Schröter und Rosanes behandelten Fragen stehem, in so allgemeiner form wohl noch nicht ansgesprochen sind, und die

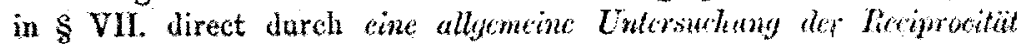
bestätigt werden.

Die im Vorigen entwiclielten Begrife gestatteten, die Traisformation einer (gewöhnlichen) Flüche aweiter Grades von einem nenen Gexichtspunkte aus zu behandeln. Endlich ist fol letzen l'aragraphen die Transformation gegebener linearer Formen untersuclit, insbesondere die Hermite'sche Transformation eines Kegelshnittes in Kaume entwickelt.

$\$ 1$.

Die orthogonale Substitution.

Eine orthoyonale lineare Substatution bei $n$ homogenen Varialoeln:

$$
y_{i}=\sum_{k} a_{i k} x_{k}
$$


ist dadorch charakterisirt, dass

$$
\sum y_{i}^{2}=m \sum x_{i}^{2}
$$

ist. Demnach müssen bekanntlich die Gleichungen:

$$
\sum a_{i k} a_{i l}=0, \quad \sum_{i} a_{i k} a_{i k}=m
$$

stattfinden. In Folge dessen lassen sich die Gleichungen (1) umkehren, so dazs:

$$
m x_{i}=\sum_{k} y_{k} a_{k i}
$$

womit dann auch:

$$
\sum_{i} a_{k i} a_{i i}=0, \quad \sum_{i} a_{k i} a_{k i}=m
$$

wird. Die Gleichnngen (3) oder (5) ergeben für das Quadrat der Substitutionsdeterminante:

die Gleichang:

$$
\Delta=\left|a_{i k}\right|,
$$$$
\Delta^{2}=m^{2} \text {. }
$$

Mithin hat $\Delta$ einen der beiden Werthe $\pm \sqrt{ } m^{n}$. Wir unterscheiden darnach eigentliche und uneigentliche Substitutionen. Beide spielen bei geradem $n$, welchen Fall wir vorcugsweise behandeln werden, eine wesentlich verschiedene Rolle, insofern dann eine eigentliche Substitution durch keine blosse Zeichenänderung in eine uneigentliche verwandelt werden kann. Die Wiederholung zweier Substitutionen derselben Art liefert immer eine eigentliche Substitution.

Die Elemente $x_{i}$, welche aturch die Sulstitution (1) in sich sellst ïbergehen, sind bestimmt durch die Gleichungen:

$$
y_{i}=e x_{i}=\sum a_{i k} x_{k},
$$

d. h. durch die Wurzelu der Gleichung $n^{\text {ten }}$ Grades:

oder:

$$
\left|a_{i k}-\rho\right|=0 \text {, }
$$

$$
\Omega(\rho)=0 \text {, }
$$

in welcher $\rho$ nur mit den Gliedern der Hauptdiagonale verbunden ist.

Durch Austihrung der Multiplication beweist man leicht die Relation:*)

$$
\Delta \Omega(\varrho)=(-n)^{n} e^{n} \Omega\left(\frac{m}{\rho}\right)
$$

Aus (8) folgt die Reciproeität der Wurzeln der Gleichung (7).

*) Brioschi, Liouvillè J. XIX, $\$ 53$; Fa de Bruno, ebenda pag. 320 . 
Für gerades $n$ ersieht man ferner, dass, weun $\Delta=-\sqrt{ } w^{n}$, awei Warzeln derselben gleich $t l^{\prime} m$ sind. Für ungerades $"$ dagegen ist. die Wurzel $+\sqrt{m}$ vorhanden, wenn $\Delta=m^{2}$, die Wurzel $-\sqrt{m}$ im entgegengesetzten Falle*). Wir nemen diese Wurzeln ausgezeichich oder Lesondere Wurzeln von $\Omega(\rho)=0$.

Die nicht ausgezeichneten Wurzelwerthe werden wir immer durch die Buchstaben:

bezeichuen, so dass

$$
r_{1}, p_{1}, r_{2}, p_{2}, r_{3}, \theta_{3}, \cdots
$$

$$
r_{s} a_{s}=n i \text {. }
$$

Man betrachte nun irgend zwei Lösungssysteme von $(0) \alpha_{i}, \beta_{i}$, Welchè zu den Wurzeln $\rho_{\alpha}, \rho_{s}$ gehören. Daun ergiebt sich:

$$
\begin{gathered}
\rho_{\alpha}^{2} \sum \alpha_{i}{ }^{2}=m \sum \alpha_{i}^{2}, \\
\rho_{\alpha} \rho_{\beta} \sum\left(\alpha_{i} \beta_{i}\right)=m \sum\left(\alpha_{i} \beta_{i}\right) .
\end{gathered}
$$

Daraus folut: Solald o nicht einen der besonderen Werike $\pm \sqrt{m}$ besitzt, ist $\sum a_{i}{ }^{2}=0$, forner besteht zwischon je zwei Lüsungssystemen, welche zu nicht reciprolon Wurzeln gehüren, die incolutorische (harmonische) Bezichang:

$$
\sum \alpha_{i} \beta_{i}=0
$$

Wir werden im Folgenden die Iösungen, welche zu der Wurzel $r_{*}$ gehören, durch:

$$
x_{1}^{*}, x_{z}^{*}, \cdots x_{n}^{*},
$$

die zur reciproken $\rho_{s}$ gehörigen durch:

bezeichneu.

$$
y_{1}^{*}, y_{z}^{*}, \cdots y_{k}^{*} \text {, }
$$

Indem wir uns der Bezeichnung $(\alpha \beta),\left(\alpha^{2}\right)$ an Stelle von $\sum \alpha_{i} \beta_{i}$, $\sum a_{i}{ }^{2}$ bedienen, gelten daher zwischen diesen Systemen die Beziehungen:

$$
\left(x^{x} x^{2}\right)=0, \quad\left(x^{3} y^{2}\right)=0, \quad\left(y^{x} y^{2}\right)=0, \quad\left(x^{*} y^{*}\right)=1 .
$$

Sieht man die Variabelen $x$ aberhaupt als Punkte (Elemente) eines Raumes (Gebietes) von $n-$ I Dimensionen an, so hann man sagen, dic Fliche:

$$
f=\sum x_{i}^{2}=0
$$

sei auf ein Cuordinatersisytem bezogen, dessen Ecken zut je zweim harmonische Pole dersellen bilden. Den Substitutionsprocess kana man 
als Beroeging des Ranmes bezeichnen, wern die Substitution eine eigentliche ist, im anderen Falle als eine symmetrische Umformung*) Setat man ferner:

so ist:

$$
\begin{aligned}
& y_{i}=\sum a_{i k} x_{k}, \\
& z_{i}=\sum a_{i l}{ }_{i},
\end{aligned}
$$

$$
(y z)=m(x \xi) \text {. }
$$

Die Substitution hat also die Eigenschaft, jedes Polareck der Fläche in ein anderes überzuführen, während $f$ in sich selbst übergeht. Die Coefficienten $a_{i k}$ bilden nach (3) und (5) selbst die Coordinaten zweier Polarecke. Diese letzteren gehen freilich durch die ortbogonale Substitution (1) nicht in einauder über. Aber durch zweimalige Wiederholung der nämlichen Substitution (1) leitet man die eigentliche Snb. stitution:

her, wo:

$$
y_{i}=\sum b_{i k} x_{k}
$$

$$
b_{i k}=\sum a_{i l} a_{k k}
$$

Daraus ergiebt sich fïr $x_{i}=a_{m b}$ :

$$
y_{i}=m a_{i m} .
$$

Jene beiden Polarecke können daher immer durch eine eigentliche Substitution in einander ibergefüht werden, $d$. h. sie sind als einander direct congruent $z$ u betrachten.

Andererseits ist in:

für $x_{k}=a_{m k}$

$$
y_{i}=\sum a_{i k} x_{k}
$$

$$
y_{i}=\sum a_{i k} a_{t a k}=m[i m]
$$

wo $[\mathrm{im}]$ gleich 0 oder 1 , jenachdem $i$ und $m$ verschieden oder gleich sind; so dass die gegebene Substitution das eine der beiden Polarecke in das Fundamentalgebilde der Coordinatenbestimmung überführt.

Nach (6) und (7) bleilen im Allgemeinen $n$ Punhte fest bei der Sulbstitution. Sie bilden bei geralem $n$ und eigentlichor Substitution die Folen eines van Erzeugenden der Fläke gebildeten Polygons, bei uneigentlicluer Substitation befinden sich dagegen unter ibnen zwei harmonische Pole derselben, welche nit den anderen $n-2$ Punkten verbunden Tangenten der Fläche liefern ${ }^{* *}$ ). Bei ungeradem $n$ ist

- Für ungerades $n$ würden die Bewegungen noch auf eine andere Weise von den symmetrischen Unformangen zu tremen seiu. Vgl. Klein, Math. Ann. IV, pag. 602 .

*) Frahm, Inanguraldissertation, \$15. 
mindestens immer ein nicht auf der Fläche gelegener Punt festbleibend.

Wir betrachten ferner das Product der beiden Determinanten:

$$
\Omega(+e) \times \Omega(-e) \text {. }
$$

Man erhält durch Ausführung der Multiplication, abgesehen rou dem Factor $\varrho^{*}$, eine Determinante, deren Glieder die $a_{i k}-a_{k i}$ sind, während die Glieder der Hauptdiagonale sämmtfich gleich ${ }_{e}^{\text {th }}-\rho$ sind. Das heisst: Bei geradem $n$ und tupeigentlicher Substitution nerschwindet die schicfe Determinante der $a_{i k}-a_{4 i}$ sanmt ihren ersten Onteq determinanten. (Bei ungeraden $n$ verschwindet sie natürlich identiseh.)

Man kann diesem Verschwinden der Unterdeterminanten in dem gedachteu Falle leicht eine geometrische Bedeutung anterlegen, Führt man die nämliche Substitution (1) zweimal hinter finauder uus, so erhält man zur Bestimmung der festgebliebenen Punkte die Gleichung $n^{\text {ina }}$ Grades:

$$
0 a_{k s}-m a_{k} !=0 \text {. }
$$

Bei der uneigentlichen Substitution hat dieselbe eine Dopplwurcel $\rho=m$, welche das Vorhandensein einer ganzen Reihe von Punkten anzeigt, die in sich selust übergehen. Es ist die lineare Reihe, welche die beiden nicht auf der Fhache gelegenen Punkte verbindet. Dic uneigentliche Substitution zueimul hintereinunder atsgefulht, liefert also keine cigentliche Sulstitution von allgomeinem Charaliter, sombern eine speciclle Bewegung.

Wir untersuchen noch die symmetrisele Determinante der ytudratischen Forne:

Man erhält leicht:

$$
\sum a_{i 1} x_{i} a_{k}
$$

wo:

und :

$$
(\Omega Q)^{2}=\Psi(\sigma) 2^{n}(-1)^{n} Q^{*}
$$

$$
\Psi(\sigma)=\begin{array}{cccc}
a_{11}-\sigma & a_{12}+a_{22} & \ldots & a_{1 n}+a_{\infty 1} \\
& 2 & \ldots & 2 \\
a_{n 1}+a_{1 n} & \cdot & \ldots & \vdots \\
2 & & \ldots & a_{n n}-\sigma
\end{array}
$$

$$
a=\frac{e^{2}+m}{20}
$$

Bei eigenthicher Transformation und geranen $n$ ist also $\psi$ das Quadrat eines Polynoms in $\sigma$, da je zwei reciproke Wurceln auf denselben Werth von o führen. Es lässt sich lejcht zeigen, dass $\Psi$ für jode seinor Dopquelourzeln sammat allen orsten Dnterideterminanten versoluwindat.

*) Zwei solche eigentliche Sabstitutionen ergeben dagegen combinirt eine nicht weiter ausgezeichnete eigentliche Subrtitution. 
Ist थ. B.:

so folgt:

$$
a_{u} a_{i}=\sum a_{i k} a_{k}
$$

d. h.:

$$
\stackrel{m}{u_{u}} a_{i}=\sum a_{1 ;} a_{k}
$$

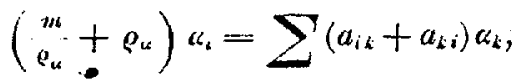

$$
\begin{aligned}
& \left(\cdots_{e_{i}}^{m}+e_{i j}\right) \beta_{i}=\sum\left(a_{i k}+a_{k i} j \beta_{k},\right.
\end{aligned}
$$

woraus ersichtlich ist, dass die Gleichungen:

$$
2 \sigma x_{i}=\sum\left(a_{i k}+a_{k i}\right) x_{k}
$$

eine lineare Reihe von Auflösungen besitzen. Die quadratische Form hat hieruach bei geradem $n$ und eigentlicher Substitution den Charakter:

$$
\text { [(11) (11) (11)...], }
$$

sie ist eine von denen, welche durch die Substitution in sich selbst übergeführt werden.

\section{$\$$ II.}

\section{Systeme von Unterdeterminanten.}

In vorigen $\$$ ist vorausgesetct, dass die Substitutionen von allgemeinstem Charakter, d. b. sämmtliche Wurzeln vou $Q(o)=0$ von einander verschieden sind. Die speciellen Fälle orthogonaler Substitutionen sind dadurch charakterisirt, dass von den $"$ Wurzeln irgend welche zusammenfallen, und stuten sich nach der Art und Weise ab, wie die Unterdeterminanten von $\Omega(\rho)$ für solche vielfache Wurzelu sich verhalten.

Man kanu die orthogonalen Substitutionen daher sach der Bescbaffeuheit der Elementartheiler von $\Omega(\rho)$ classificiren, issofern mit jeder besonderen Fertheilung derselben eine wesentliche Gruppiruny der foslblribenden Elcmente verbunden ist. Dabei zeigt sich, daws das Auftreten der Elementartheiler an gewisse Gesetze gebumlen ist, welche die Zahl der möglichen Fülle a priori auf eine gewisse lileinste Zahl einzuschrünken collublen, deren Existenz dann freilich noch einer directen Prüfung unterliegen muss. Diese Gesetze ergeben sich durch eine grenauere Betrachtung der Unterdeterminanten von $\Omega(\rho)$, die wir im Folgenden ausführen werden.

Wir betracliten die $k$-fach mit beliebigen Grössenreihen $u, v$, $w, \cdots$ vertical und $U, l, W, \cdots$ horizontal geränderte Determinante: 


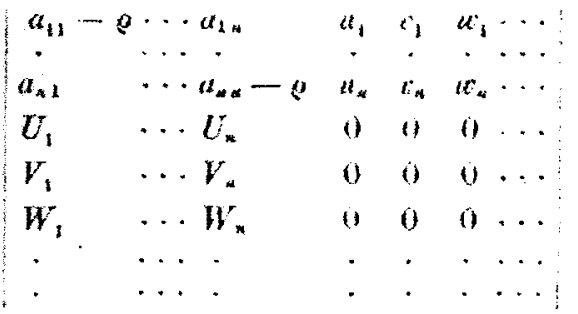

welche zur Abkürzung durch

$$
[u v w \ldots U \boldsymbol{V} \boldsymbol{W} \ldots]_{\varphi}
$$

bezeichnet werden nöge; die Coefticienten der Verbinulungen der

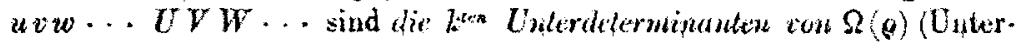
determilanten $n-k i n$ Grades).

Multiplicirt man dieselbe mit $\Delta$, so phitsteht, weun

gesetzt wird:

$$
\theta^{*}=\frac{k}{s},
$$

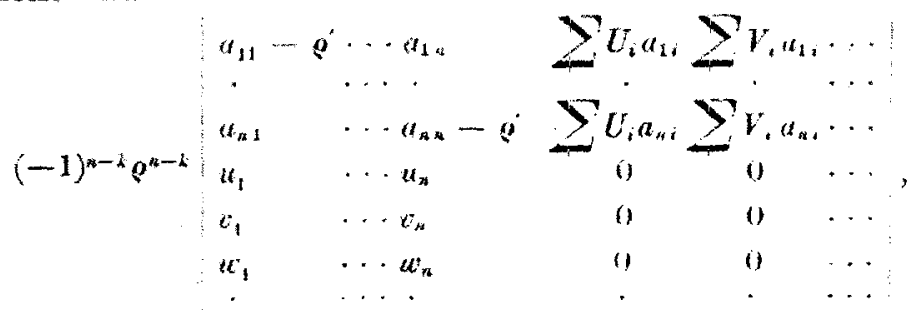

oder, wenn $\sum U_{i} a_{r}=U_{r}, \sum V_{i} a_{r} \ldots V_{r}$, die ditehung:

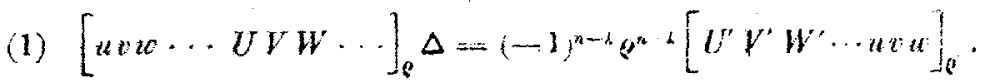

Hat demnach die linke seite dep Factor $10-a_{10} j^{m}$, so nuss die rechte: Seite den Factor $\left.\mid \rho^{\prime}-\varphi_{\alpha}\right]^{\text {th }}$ haben. Damit ist bewiesen, dass das Ver-

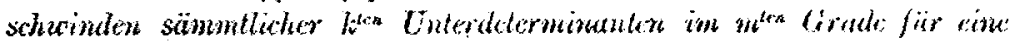

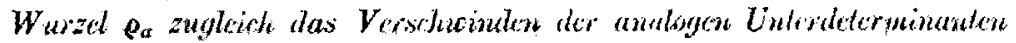

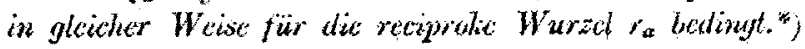

Man kann die rechte Seite der Gleichung (1) in einc neue Form bringen. Zieht man uämlich die $n$ ersten Ferticalreihen, mit den $U_{i}, V_{i}, W_{i} \ldots$ multiplicirt, vou den folgendex $a b$, so treten an stello der $U^{\prime} V^{\prime} W^{\prime} \ldots$ wieder die mit $\rho^{\prime}$ multiplicirten $l^{\prime}, V, W$ selbst, wärend die Nullen in der Ecke der Determinante durch die Ausdrucke $-(u U)_{x}-(u V)_{,}-(a W)$ etc. $z u$ ersetzen sind.

* Voss. Hathematische Ammalen X, pag. 163. 
Ein besonders bemerkenswerthes Resultat ergiebt sich hier, wenn $e= \pm \sqrt{m}$ genommen wird. In diesem Falle ist $\varrho=\varrho$. Ist zunächst $n$ gerade, $l=1$ und die Substitution eine eigentliche, so hat man aus (1) nach Ausführung der eben augegebenen Reduction:

$$
\Delta[u U]_{ \pm V^{\prime m}}=(-1)^{n-1} Q^{n-1} \varrho^{\prime}\left[[U u]_{ \pm V_{u}}-\frac{1}{e^{\prime}} \Omega( \pm V m)(u U)\right]
$$

oder, da $\Delta=\varphi^{\prime \prime}$,

$$
[u U]+|U u|_{ \pm V_{m}}=\cdots \frac{1}{ \pm V_{m}} \Omega( \pm \sqrt{m})(u U)
$$

Daraus ist, wenu wir die ersten Usterdeterminanten von $\Omega$ durch $\Delta_{i k}$ bezeichen:

$$
\Delta_{i k}=-\Delta_{k i}, \quad \Delta_{i i}=\Delta_{i k}=\mp \frac{\Omega\left( \pm V_{m}\right)}{2 V_{m}} .
$$

Dite ersten Unterdeterminunten vom $\Omega(+\sqrt{m})$ bilden also eine schiefe Determinante, deren Hauptliagonalglieder ein und denselben Worth besitzer.

Man haun also das adjuugirte System der $\left[a_{i k}+\sqrt{m}\right]$ in der form:

$$
\begin{aligned}
& \mathrm{B}=\mid \begin{array}{llll}
b_{11} & b_{12} & \cdots & b_{1 n} \\
b_{21} & b_{22} & \cdots & b_{z 4} \\
b_{n 1} & b_{n 2} & \cdots & b_{n k 4}
\end{array} \\
& b_{i k}=-b_{k i}, \quad b_{i i}=-\frac{\Omega( \pm V m)}{2 V_{m}}=\omega
\end{aligned}
$$

darstellek. Nun sind aber die ersten Unterdeterminanten des adjungirten Systemes wieder den ursprïnglichen Elementen proportional. Bezeichnet man also die Unterdeterminanten der $b_{i k}$ durch $\beta_{i k}$, so hat man:

$$
\begin{aligned}
& \beta_{i k}=-a_{i k}(\Omega(+\sqrt{m}))^{n-*}, \\
& \beta_{i i}=-\left(a_{i i}+\sqrt{m}\right)(\Omega(+\sqrt{m}))^{n-2} .
\end{aligned}
$$

Dis endich die Determiuante $B$ selbst fyleich $(\Omega(+\sqrt{m}))^{n-1}$ ist, so hat wan schliesslich:

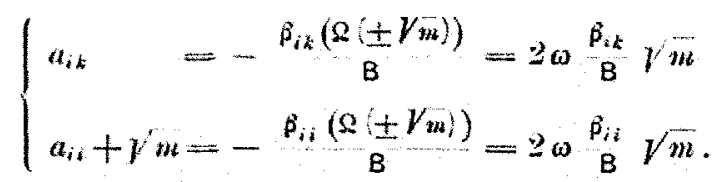

Es sind die Formeln (4) aber genau diejenigen, welche Cayley. zur bestimmung der Coefficienten einer orthogonalen Substitution angegeben hat.*) Damit ist bewiesen, dass in der 'That jene Cayley'schen 
Formeln die allgemeinsten sind, aclche dise Ayfgatre lisen, eine Frage, welche bisher eine Läcke in der 'Theorie def orthogonalen Substitutionen bildete. Auch Herr Rosanes*) hat schon gelegentlich bemerkt, liass aus dem Umstande, dass die Cayley'schen Formeln die erforderliche Zahl willkürlicher Parameter enthalten, noch nicht geschlossen werden könne, dass sie deh vollen (Grad der Allgemeinheit besitzen; ;*) es lasse sich aber ohne schwierigkeit ein befriedigender Beweis dafür erbringen.*:) Immerhin dürfte der vorhin gegebene, welcher direct die Coefficienten $a_{i k}$ als Unterdeterminanten einer schiefen Determinante nachweist, einfach und übersichtlich genug sein, um hier eine Stelle zu beanspruchen.

Für eine uneigentliche Substitution verschwindet dagegen bei geradem $n$ die Determinante $\Omega( \pm \sqrt[V]{ } \dot{w})$; die Gleichung (1) gitebt dann:

$$
\Delta_{i k}=\Delta_{k i}
$$

d. h.: die ersten Unterdetchinanten bilden ein symmetrisedus Syskm, so dass die Lösung $a_{i}$, welche z. B. der Wurzel $+\sqrt{m}$ entspricht, der fortlaufenden Proportion grenügt:

$$
a_{1}: a_{1} \alpha_{2}: \alpha_{1} \alpha_{3}: \alpha_{1} \alpha_{4}: \cdots=\Delta_{11}: \Delta_{12}: \Delta_{1 ;}: \Delta_{i 4} \cdots
$$

* Rosanes, Crelle 80, 1. 52, 71.

**) Vgl. dazu $\$ V$., wo in der That sulatitutionsformeln mil vicl wehr will. kurlichen Parametern angegeben sind, die keinesweg allomein sind.

** Wir heben noch folgenden Zaeammenaug zwischen den Coefticieutcu $a_{i k}$ und $b_{i k}$ hervor. Wenn nach Cayley:

und

$$
a_{i k}=-\beta_{, \alpha}{ }_{\mathrm{B}}^{\omega} \gamma m-[\gamma m]
$$

ist, so folgt:

$$
2 a_{i k} x_{k}=x_{i}
$$

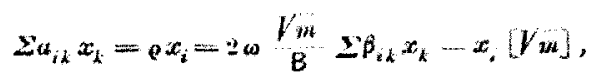

also wenn mit $b_{i j}$ multiplicirt wird, wegen $2 b_{i k} \beta_{i k}-\mathrm{B}$,

$$
\left(e+\gamma n \geq x_{i} b_{i j}-2 \omega \gamma n x_{j}\right.
$$

Hiernach findet zwischen den Wurzeln der Determinanten $g$ ro und $b_{2 i}-e^{x}$ der Zusanmenhutog statt, daso

$$
e=\frac{2 \omega \sqrt{m}}{V w+\varphi^{\prime}}
$$

wogeren die ersten Uazerdeterwinanten beider Getermianten für correbpondireode Wurzelwerthe proportional sind. Also ist mit joder endicher eigenthichen ordho. gonalen Substitation $y_{i}=\sum a_{i k} x_{k}$ cine wiendhich kleine:

$$
y_{i}=x_{i}+\left(\geq b_{i t} x_{i}\right) d t
$$

verknitpt, welche dasselbe Gebilde von $n$ Punkten angeändert läst, deren Wieder. holung aber nicht die endliche Substitation nervorbringt. 
Wir setzen vorads, es seien die $k-1^{\text {tev }}$ Unterdeterminanten die letzten, wekhe sämmilich für eine Wurzel $r_{\alpha}$ verschwinden. Man kann dann in (1) die Summen - $(u U)$, weil sie keine Beiträge zu den. Werthen der $7^{\text {ten }}$ Unterdeterminanten liefern, fortlassen, and erhält so:

(5) $\Delta[u v w \cdots U V W \cdots]_{Q}=(-1)^{n-k} e^{n-k} Q^{k}[U V W \cdots u v w \cdots]_{i^{\circ}}$.

Bezeichnet man nun, wie gebräuchlich, den $2 k^{\text {ten }}$ partiellen Differentialquotienten:

durch:

$$
\frac{\partial^{2 k}}{\partial u_{i} \partial v_{k} \partial w_{i} \cdots \partial U_{j} \partial V_{x} \partial W_{2} \cdots}
$$

so hat man:

$$
\Delta_{i 26, \ldots j \times 2 \ldots,}
$$

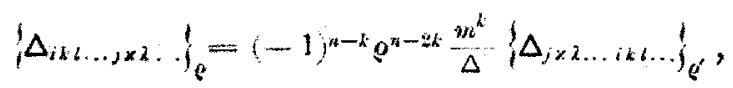

eine Gleichung, nach welcher die Unterdeterminanten reciproker Wurzeln gewisse Symmetrieeigenschaften besitzen. Wir wollen dieselbe zunächst für den Fall in Anwendung briugen, dass bei eigentlicher Substition $\pm \sqrt{m}$ eine der Wurzeln ist. Es folgt für $k=1$ :

$$
\Delta_{i k}=-\Delta_{k i}, \quad \Delta_{i i}=-\Delta_{i i}=0 .
$$

Die bekannte Identität zwischen den ersten Unterdeterminanten einer versehwindenden Determinante:

$$
\Delta_{i i} \Delta_{k i}-\Delta_{i k} \Delta_{k i}=0
$$

zeigt dann, dass auch $\Delta_{i k}=0$. Die ausgezeichneten Wurzeluerthe bedingen daher, wenn sie nicht, wie bei geradem $n$ und uneigentlicher Substitution, oder bei ungeralem $n$, eo ipso der Transformation zukommen, mindestens das Verscliwinden aller arsten Unterdeterminanten.

Mau kann diesen Schluss erweitern. Wir nehmen an, dass nicht allein die ersten, sondern woch die $2 m^{\text {leis }}=k_{1}^{\text {tei }}$ Unterdeterminanten sämmtlich versehwinden für einen dieser ausgezeichneten Werthe. In diesem Falle ist bei eigentlicher Substitution:

$$
\Delta_{i k 3 \ldots ; \times \lambda \ldots}=-\Delta_{j \times \lambda ., i k \ell \ldots,}
$$

woraus zanächst das Verschwinden aller $l^{* * n}$ Hauptunterdeterminanten, danit aber das Verschwinden aller $h^{\text {iva }}$ Unterdeterminanten folgt. Verschuinden also bei einor cigentlichen Substitution fïr $\varrho= \pm \sqrt{m}$ noch die sämmatlichen $k^{\text {tew }}$ Unterdetorminanten, die höheren dagogen wicht, so wit $k$ nothucendig eine ungerade Zuhl.

Ein aualoges Theorem läst sich auch für uneigentliche Substitutionen aufstelleu. Weun hier $k-1=2 m-1$ ist, so hat man wieder für $\rho= \pm 1 m$

$$
\Delta_{i k k \ldots}=-\Delta_{j \times 2 \ldots,}
$$


so dass bei uneigentlicher Substilation immer uar die sümmitlichen Vuter-

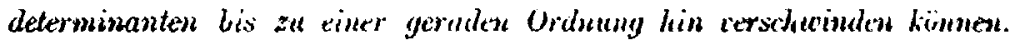

Dass das Verschwinden der $k^{\text {two }}$ Hauptunterdeterniuanten dus sämmtlicher $k^{\text {ten }}$ linterdeterminanten nach sich zielst, beruht auf einem allgemeinen Satze, dessen Beweis wir bier yoch besonders einschalten. Derselbe lautet:

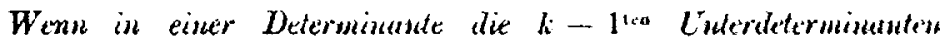
sämmtlich ierschuinden, dagegesi die $k^{\text {tron }}$ Unterdeterminanten cin symmetrisches oder alterniretudes system bilden, so kïmuen "lisse ktzkro" nur dann von Null verschicilen sein, wenn sich unter den Hauptunter. determinanten nicht revschwindende befisuden, and umgelicht zicht das Verschuinden der tikn Hauplenterdeterminamlen das aller anderen nach sich.

Ein etwas allgemeinerer Satz ist neuerdiugs von Herrn Frubenius*) ausgesprochen worden. Zun Beweise bedienen wir uns ähnlicher Ueberlegungen, wie sie zur Berrrüudung des hroneckerschen Satzes**) in Anwendung kumuen, führen ihn aber der Uelersichtlichkeit halber an einer sechsreihigen Determinante der $b_{i \hbar}$, deren vierreihige Unter. deterninanten sämutlich, deren dreireitigge Hauptunterdeterminauteu ebenfalls verschwinden.

Sei nun eine der nicht verschwindenden dreireihigen Determininten:

$$
S=\begin{array}{lll}
b_{14} & b_{1,} & b_{16} \\
b_{24} & b_{3:} & b_{3,} \\
b_{31} & b_{3,} & b_{36}
\end{array}
$$

so folgt aus dem identischen Verschwinden von:

$$
\begin{aligned}
& b_{1} b_{1,} \|_{1,} \sum b_{1}, \mathrm{~B}
\end{aligned}
$$

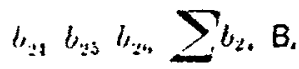

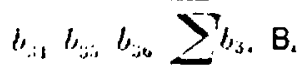

$$
\begin{aligned}
& b_{a}, b_{a b} b_{\alpha i j} \sum b_{a}, B
\end{aligned}
$$

für $B_{1}=0, B_{i}=0, B_{i j}=0$, nenn die $B_{1}, B_{i}, B_{i}$ die Gileichungen:

$$
\sum b_{1}, B_{1}-0, \quad \sum b_{2,} B_{1}=0, \quad \sum b_{3} B_{1}-0
$$

befriedigen, dass auch:

$$
\sum b_{u i} B_{i}=0
$$

Demnach verschwinden alle dreireibigen Determinanten: 


$$
\left|\begin{array}{lll}
b_{a 1} & b_{c 2} & b_{\alpha 3} \\
b_{\beta 1} & b_{\beta 2} & b_{\beta 3} \\
b_{\gamma 1} & b_{\gamma 2} & b_{\gamma 3}
\end{array}\right|
$$

Unter diesen befiudet sich aber nach der Voraussetzung dem absoluten Werth uach einnal auch die Determinante $S$, womit wir auf den Widersprueh $S=0$ gefuhrt sind. Das Verselwinden einer dreireiligien Hauptuviterdaterminante zielht also das aller derjenigen dreireihigen Determinanten nach sich, die aus den Horizontalreihen und Verticalreiken gebildet werden liönnen, die sidh in jener liveuzon.

Man kann jenes Verschwinden der Unterdeterminanten auch mit den Betrachtungen über schiefe Determinanten in Verbindung bringen. Wenu man die Determinante

nit

$$
[a v w \cdots U V W \cdots]_{+\varepsilon}
$$

$$
\Omega(-p)
$$

multiplicirt, so entsteht eine Determinante, dereu $k$ Ränder, so lange $\Omega(-\varrho)$ nicht selbst verschwindet, wieder als aus ganz beliebigen Grösseu bestehend angesehen werden können, deren Kern aber eine schiefe Determinante bildet, deren Diagonalglieder für $\varrho= \pm \sqrt{m}$ sämmtlich Null sind. Dann folgt der obige Satz leicht aus dem Theorem, dass in einer schiefen Determinante (gerader Ordnung) die Reihe der verschwindenden Uuterdeterminanten immer mit einer uugeraden Zahl schliesst.") Aber es erscheint zweifelhaft, ob dieser Beweis eine Ausdehunng auch auf diejenigen lialle gestattet, in denen $\Omega(-\rho)$ ebenfalls gleich Null ist.

Das Verschwinden der Hauptunterdeterminauten, welches aus Gileichung (6) sich ergab, kann noch auf andere Art dargethan werden, welche bier kurı angedeutet werden möge.

Setat man die gegebene eigentliche (uneigentliche) Substitution mit einer ungeraden (geraden) Zahl $k$ uneigentlicher von der Art:

$$
\left.y_{i}=x_{i}-2 a_{z} a_{i}^{* *}\right)
$$

cusammen, so entsteht eine uneigentliche Substitution, d. h. eine solche, deren Determinante $\Omega(\rho)$ die beiden $W$ urzeln $\pm \sqrt{m}$ besitzt.

Daraus ergielbt sich der Satz:

Rändert man die Determinante der $a_{i k}-\rho \mid$ rertical mit $k$ belicbigen Grössenreihen $a_{i}, b_{i}, c_{i} \cdots$, und horizontal mit denselben, aber mit dem Foctor o multiplicirt, wod ersetat man noch die $k^{2}$ Elcmente der Ecke aured ans Suluma:

* Frobenius, Crelle $\Varangle 2$, pag. \$4:

**) Vgl aber aolche Substitutionen noch besonders $\lessgtr V$. 


$$
\begin{array}{cccc}
-1 & -2(a b & -2(a c) & \cdots \\
0 & -1 & -2(b c) & \cdots \\
0 & 0 & -1 & \cdots \\
. & . & . &
\end{array}
$$

so entsteht eine Determinante, welche für $\omega_{-}= \pm \sqrt{\text { m identisch Noll }}$ ist, welches anch die Werthe der $a, b, c \ldots$ sind.

Nimmt man alle a mit Ausnahme eines einzigen, welches gleich 1 gesetzt wird, gleich Null, eberso alle $b, c, \cdots$, mit Ausnabme eines eivaigen gleich Null, so ergeben sich Gleichnngen, welche eine

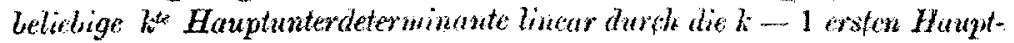
wnterdeteminantensysteme ausdriekn, womit der Beweis des Satzes geliefert ist.

Zum Schluss fügen wir noch hinzu:

Findet das Verschwinden der lece Unterdeterminanten statt, so existirt imner, eine $h$ fache Mannigfaltigkeit von Lösungen der Gileichungen (1). Dieselben stellen sämmtlich Punkte von $f=0$ vor, wenn die Wurzel, für welche das genaunte $\rho$ stattindet, nicht einen der Werthe $\pm \sqrt{m}$ besitzt. Die involutorische Bezielung bleibt dabei fin alle nicht reciproken Wurzeln angehörigen Lözungen erhalten. Die allgemeine Lüsing der Gleichnugen (1) ist also für die Wurzel $\rho_{\text {ix }}$ von der Form:

$$
\tilde{z}_{i}=\sum_{i}^{+1} z_{i}^{k} \lambda_{k}
$$

mit der Eigenschaft $\sum_{z_{i}}=0$, welches auch die Werthe der will. kürhichen Constanten $\lambda_{k}$ sind.*)

s III.

Formen, weleho dureh dio Substitution in sich ubergehen.

Nach der obigen Bezcichnong war:

oder:

$$
r_{i} x_{i}^{*}=\sum a_{i k} x_{k}^{4}, \quad \text { e. } y_{i}^{*}=\sum \prime_{i k} y_{k}^{*},
$$

$$
\left\{\begin{array}{l}
r_{*} \sum y_{i} x_{i}^{*}=m \sum x_{i} x_{i}^{*}, \\
0_{*} \sum y_{i} y_{i}^{*}=n \sum x_{i} y_{i}^{*} .
\end{array}\right.
$$

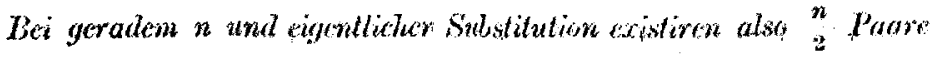
con linewen Formon, welde, in sich tratsformint werden, ste stellan $n$ 
feste Tangentialebenen der Flüche vor. Wir bezeichnen sie der Reihe nach durch:

so dass:

$$
\left\{\begin{array}{l}
X_{1} X_{2} \ldots X_{s}, \\
\equiv_{1} \Xi_{2} \ldots \Xi_{s}, \quad(2 s=n)
\end{array}\right.
$$

Fur die $X_{i}, \equiv_{i}$ besteht die Identität:

$$
X_{r}=\sum x_{i} x_{i}^{r}, \quad \equiv,=x_{i} y_{i}^{r}
$$

Sie ergiebt sich durch Multiplication der verschwindenden Determinante:

$$
\begin{aligned}
& x_{1}^{\prime} \cdots x_{n}^{\prime} \quad X_{1} \\
& \dot{y_{1}^{3}} \cdots \dot{y_{3}} \equiv \\
& x_{1} \ldots x_{\mu} \quad \sum x_{i}^{2}
\end{aligned}
$$

mit der Determinante der $x_{i}^{\prime}, y_{i}^{*}$. Die directe Ausführung der rechten Seite von (4) liefert dagegen:

so dass:

$$
\sum X_{i} \equiv_{i}=\sum x_{t} x_{t}^{i} y_{s}^{i} x_{s}=\sum x_{t} x_{s}\left(x_{t}^{i} y_{s}^{i}+x_{s}^{i} y_{t}^{i}\right)
$$

$$
2 \sum x_{t}^{i} y_{t}^{i}=1, \quad \sum x_{t}^{i} y_{s}^{i}+x_{s}^{i} y_{t}^{i}=0
$$

Den Gleichungen (1) zufolge gehen nicht nur die linearen Formen $X, \equiv$ in sich über, soudern auch jede homogene Function der Producte:

$$
\left(X_{r} \Xi_{r}\right) \text {. }
$$

Insbesondere sind die qualratiscken Formen dieser Eigenschaft ron der Gestalt:

$$
F=\sum X_{i} \equiv_{i} \lambda_{i} * j
$$

Unter den Formen (6) befinden sicb auch speciclle, welche der Gleichung:

$$
\sum\left(\begin{array}{c}
d F \\
x_{i}
\end{array}\right)^{2}=\mathrm{M} \sum x_{i}^{2}
$$

genügen. Sie entsteben, wenn man den Coefficienten $\lambda$ die Werthe \pm 1 in beliebiger Abwechselung ertheilt. Werden von den $s=\pi / 2$ Grössen $\lambda k$ gleich +1 , die übrigen $l$ gleich -1 angenommen, so ist die Charakteristik der Deterninante derselben:

-) Dass diese Formen die einzigen sind, welche in sich üuergehen, erkenut man, wenn man an Stelle der Variabelen $x_{i}$ einer quadratischen Form $\sum x_{i} x_{k} a_{i k}$ die $X_{i}, \equiv_{i}$ einführt. 


$$
\left.\left.[111 \cdots)_{2}(1),\right)_{1}\right] \text {, }
$$

sie stellen daher nur dann Formen ron allgemeinstem Charakter vor, die der Gleichang (7) genügen, wenn $n$ von der Form $4 m$ ist')

In Algemeinen können bei eigenthicher Substitation keine linearen Formen gegenseitig in einander übergelsen.

Denn ans:

folgt:

$$
\begin{aligned}
& \sum \alpha_{i} a_{i k}=\rho \beta_{k}, \\
& \sum \beta_{i} a_{i k}=m \alpha_{k}
\end{aligned}
$$

$$
\sum \beta_{i}\left(a_{i k}-e a_{k i}\right)=0 \text {, }
$$

so dass die Coefficienten einer solchen Form $\beta_{x}$, welche durch die Substitution gegenseitig mit $\alpha_{*}$ veriauscht wird, den ersten Unterdeterminanten der verschwindenden Determinante:

$$
\left|a_{i s}-p a_{b i}\right|=0 \text {, }
$$

proportional sind. Aber aus den Gleichungen:

folgt:

$$
\begin{aligned}
& \sum a_{k} x_{k}^{*}=x_{k}^{*}, \\
& \sum a_{k} x_{k}^{*}=b_{k} x_{i},
\end{aligned}
$$

$$
a_{k i}-a_{k i} \frac{r_{s}}{\theta_{s}}=0 \text {. }
$$

D. h.: dio Worzels der Gkichung (8) sind sämbtlich reciprok whd glech den Ferhältwissen der netipolien Wurzeln der Gleichung $\Omega(\rho) \neq 0$,

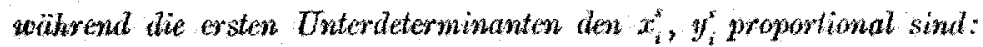

Sehreibt man (9) in der form:

$$
a_{i k}-a_{i i} \frac{p^{* k}}{m z}=0 \text {, }
$$

so erkennt man, dass die sämmitichen erstem Unterdeterminanton non

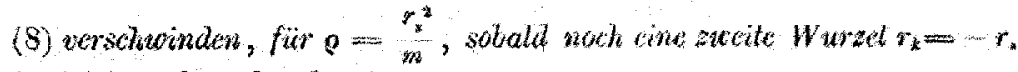
in $\Omega(\rho)=0$ vorhanden ist.

In diesem letateren Falle gelen die linoun Büsched $X_{*}-\tilde{k} X_{*}$,

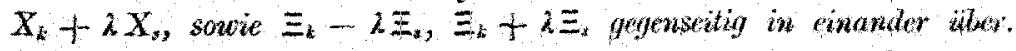
Zugleich gehen auch die Producte $X_{i} \Xi_{s}, X_{s} \Xi_{k}$ in $-\frac{1}{n} X_{k} \Xi_{x}$ $1 X_{s} \equiv_{k}$, mithin die quadratischen formen:

$$
X_{k} \equiv_{s} \lambda_{k}+X_{k} \Xi_{k} \lambda_{k}
$$

in sich uber. Enthalt insbesondere die Determinante $2(0)$ nur Potenzen

* Fuss, Matherwatische Awiaten $X, p y z$.117. 
von $\rho$ mit geraden Exponenten, so ist die Determinante (8) das Quadrat einer ganzen rationalen Function von $\rho$, und die quadratischen Formen mit $n$ Parameteru:

$$
\sum X_{k} \equiv_{s} \lambda_{k}+X_{s} \Xi_{k} \lambda_{s}
$$

gehen in sich uber. Sie geniigen der Differentialgleichung (7), wenn jedes Parameterpaar der Gleichung:

$$
\lambda_{k} \lambda_{a}=\text { constans }
$$

genügt, und besitzen dann eine Determinantenchavakteristik, welche von allgemeinstem Charakter ist, d. h. lauter einfache Elementartheiler hat*).

Bei einer uneigentlichen Sulstitution dugegen existiren zwei besondere Formen $X_{1}, \equiv_{1}$, welche keine Tangentinlebenen der Fläche vorstellen. In Folge dessen findet die Identitait:

$$
\sum x_{i}^{2}=X_{1}^{2}+\equiv_{1}^{2}+2 \sum x_{k} \equiv_{k}
$$

statt, und die quadratischen Formen:

$$
F=\lambda_{1} X_{1}{ }^{2}+\mu_{1} \equiv_{1}^{2}+\sum X_{k} \equiv_{k} \lambda_{k}
$$

gehen in sich uber. Die Fornien, welche der Gleichung (7) genügen, entatehen, wenn man wieder die Coefficienten $\lambda, \mu$ gleich +1 setzt. Insbesondere sind die beiden quadratischen Formen:

$$
\left\{\begin{array}{r}
X_{1}{ }^{2}-\Xi_{1}{ }^{2}+2 \sum\left(X_{k} \equiv_{k}-X_{l} \equiv_{i}\right) \\
-X_{1}{ }^{2}+\Xi_{1}{ }^{2}+2 \sum\left(X_{k} \equiv_{k}-X_{i} \equiv_{i}\right)
\end{array}\right.
$$

zu erwähnen, welche die allgemeinste Determinantencharakteristik besitzen, sobald $k=l$ sein kann, $d$. h. wenn $n-2$ durch 4 theilbar ist.

Die Schaar quadratischer Formen:

$$
\lambda_{1} X_{1}{ }^{2}+\mu \equiv_{1}^{2} \pm 2 \lambda X_{1} \equiv_{1}+2 \sum X_{k} \equiv_{k} \lambda_{k}
$$

dagegen besitzt die Eigensehaft, lass je zwei entgegengesetztem Zeichen von $\lambda$ entsprechende Formen gegenseitig in sich ïbergehen. Auch unter ihnen befinden sich specielle Formen, welche der Gleichung (7) genugen; sie entstehen, wenn man $\lambda_{1}=\sqrt{1-\lambda^{2}}, \mu=-\sqrt{1-\lambda^{2}}$, und die ubrigen $\lambda_{i}$ gleich \pm 1 setat, und sivd rom allgemeinsten Charakter, wenn in der Summe $\sum X_{k} \equiv_{k} \lambda_{k}$ sich gleichviel Glieder mit positivem und negativem Zeichen befinden.

*) Ein Beispiel hierfur liefert die Transformation der Flkche zweiten Grades, bei welcher die beiden Systeme der Ersengenden unter einander vertauseht werden. V.gl. \& VIII. 
§ IV.

Vertauschbare Substitationen.

Zwei Substitutionen, (a), (b):

$$
\left\{\begin{array}{l}
\text { (a) } y_{i}=\sum a_{i k} x_{k}, \\
\text { (b) } z_{j}=\sum b_{j i} x_{l},
\end{array}\right.
$$

heissen vertauschbar, wenn die Ordnung, in welcher sie nach einander ausgefuhrt werden, für das Resultat der Substitution gleichgultig ist. Dies führt auf die Bedingungen:

oder :

$$
\rho \sum a_{j i} b_{i k}=\sum b_{j i} a_{i k}
$$

$$
m \varrho b_{s k}=\sum b_{j i} a_{i k} a_{j s}
$$

Die Gleichungen (3) sagen aus, dass die bilineare Form:

$$
F\left(y y^{\prime}\right)=\sum b_{m n} y_{m}^{\prime} y_{n}
$$

vermäge der Substitution (a) in sich übergeführt wird. In der That hat man:

$$
F\left(y y^{\prime}\right)=\sum b_{m n} y_{m}^{\prime} y_{n}=\sum b_{m n} a_{m r} a_{n s} x_{r}^{\prime} x_{t}=p m F\left(x x^{\prime}\right) .
$$

Aus dem vorigen \& III. ergiebt sich aber für den Ausdruck der bilinearen Formen dieser Eigenschalt ohne Weiteres der Ausdruck:

$$
F=\sum l_{i} X_{i} \equiv_{i}+\lambda_{i} X_{i} \equiv_{i} \text {. }
$$

Und umgekehrt ergeben sich daraus als Coefficienten einer mit (a) vertauschbaren Substitution (b) die Grössen :

$$
b_{m n}=\sum\left(l_{i} x_{m}^{i} y_{n}^{i}+\lambda_{i} x_{n}^{i} y_{m}^{i}\right)
$$

Sollen die $b_{m n}$ ebenfalls Coefficienten einer orthogonalen Substitution sein, so findet man leicht nach \& III. (5), dass:

$$
\lambda_{i} l_{i}=\infty \text {, }
$$

d. h. gleich einer beliebigen Constanten zu setzen ist.

Man kann die Aufgabe, sämmutliche Systeme der $b_{m n} z \mathfrak{z}$ bestimmen, noch auf eine andere Weise lösen.

Man multiplicire die Gleichungen (3):

$$
\rho \sum a_{j i} b_{i k}=\sum b_{j i} a_{i k}
$$

mit $x_{k}^{s}$, so entsteht, wegen:

$$
r, x_{i}^{\prime}=\sum a_{i k} x_{k}^{2}
$$


Wird also:

$$
\sum b_{i k} x_{k}^{s}=y_{i}^{*}
$$

gesetat, so ist:

$$
\text { e } \sum a_{j i} \gamma_{i}^{*}=\gamma_{s} \gamma_{j}^{*}
$$

ans welcher Gleichung hervorgeht, dass $\varrho=1$ zu nehmen ist und dass die Grössen $y_{j}^{\prime}$ von den $x_{j}^{*}, y_{j}^{3}$ nar um willkürliche Factoren ververschieden sein können. Damit aher hat man zur Bestimmung der $b_{i k}$ dre Gleichungen (7) oder:

$$
\left\{\begin{array}{l}
\sum b_{i k} x_{k}^{*}=l_{s} x_{i}^{*}, \\
\sum b_{i k} y_{k}^{*}=\lambda_{s} y_{i}^{*},
\end{array}\right.
$$

aus denen wieder die Gleichungen (6) hervorgehen.

Wir haben dabei die Substitution als eigentliehe und $n$ gerade vorausgesetat. Is ist leicht, die Modificationen auzugeben, welche die abrigen Falle mit sich füliren wïrden. In dem bier betrachteten Falle ist natürlich die Substitution (b) wieder eine eigentliche, was sich auch durch Bestimmung der Determinante der $b_{i k}$ ans den Gleichungen (7) ergiebt.

$J_{e}$ zwei mit (a) vertausehbare Substitutionen (b), (B) sind num auch unter sich vertauschbar, was sich ebenfalls leicht direct verificiren lasst. Da jede Transformation (a) mit sich selbst vertausehbar ist, so muss in der Lösung (b) bei geeigneter Wahl der $l, \lambda$ auch das System der $a_{i k}$ selbst enthaiten sein. In der That kann man leicht die $a_{i k}$ dureh die $x_{i}^{*}, y_{i}^{*}$ ausdrücken und erhält dann:

$$
a_{i k}=\sum\left(x_{k}^{s} y_{i}^{a} \varrho, x_{i}^{*} y_{k}^{z} r_{s}\right)
$$

Diese Darstellung dex $a_{i k}$ sctst uns in den Stand, die allgemeinste Zerlegung der gegebenen Substitution in beliebig viele andere vertauschbare auszufuhien.

Eine beliebige Zahl von Substitutionen:

$$
\left\{\begin{array}{c}
b_{i k}^{\prime}=\sum l_{s} x_{i}^{*} y_{k}^{s}+\lambda_{s} x_{k}^{*} y_{i}^{s}, \\
b_{i k}^{*}=\sum l_{s}^{*} x_{i}^{*} y_{k}^{*}+\lambda_{k}^{*} x_{k}^{*} y_{i}^{s}, \\
\vdots \\
b_{i k}^{*}=\sum l_{s} x_{i}^{*} y_{k}^{*}+\lambda_{s}^{*} x_{k}^{*} y_{i}^{*},
\end{array}\right.
$$

setzt sich nämlich zor Transformation (10) zusammen, wenn:

$$
l_{s} T_{s} \ldots l_{s}^{r}=r_{s}
$$


genommen wird. Unter diesen Substitutionen sind $\frac{2}{2}$ kanonische besonders bemerkenswerth, welche entstehen, wean jedesmal ein System der $l, \lambda$ gleich einem Paar reciproker Wurzeln $r_{x}, \varrho_{s}$, die anderen gleich $\sqrt{m}$ angenommen werden. Es sind dies Transformationen, bei denen den $n-2$ Wurceln $\sqrt{m}$ entsprechend, ein linearer Ausdruck mit $n-2$ Parametern and wwei Pankte der Flikche fest bleiben, $a . h$. die $\frac{n}{2}$ Elementarrotationen, in welche die allgemeinste Feweging sith zerlegen lässt. Indessen gehen wir uicht weiter auf jene Zerlegung eiu, da sie von verschiedenen Seiten sowohl für beliebige Dimensionen, als auch insbesondere für den projectivischea haum von drei Dimensionen. bereits untersucht worden ist*).

Die Formeln (11) führen noch auf eine andere Zorlegung. Man setze $\frac{n}{2}$ der Zahlen $l, \lambda$ sämmtlick gleich $k$, und ordne sie einer Gruppe von $\stackrel{n}{2}$ solehen Punkten aus den $n$ bei der Transformation (10) festbleibenden Punkten $x, y \mathrm{z}$, deren Verbindungslinien Erzeugende der Flache $f=\sum x^{2}=0$ sind. Opdnet man den Werthen $\frac{\omega}{k}$ die äbrigen ${ }_{2}^{n}$ Punkte $2 u$, so entsteht eine Transformation mit don beiden

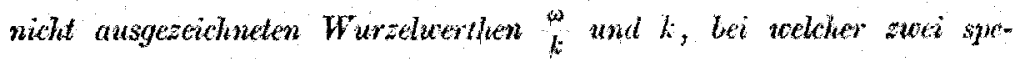

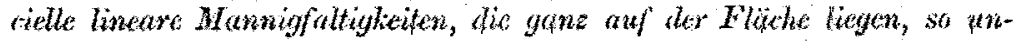
geändert bleiben, dass jeder Puntet dersellun fost beill. Wine soldie Anordnung der Grappe der $n$ festbleibeuden Punkte $x, y$ ist auf $22^{-2}$-fache Art möglich, so das im Ganzen $2^{-1}$ Traspormationen dieser Art existiren.

Betrachten wir jetat irgend eine dieser Transformationen, etwa diejenige, welche sämmtliche $x$ in eine Gruppe vereinigt, so bleiben bei derselben die folgenden beiden speciellen liquaren Aqudrüke fest:

$$
\begin{aligned}
& \xi_{i}=\sum x_{i}^{r} m_{r}, \\
& s_{i}=\sum y_{i}^{r} \mu_{r},
\end{aligned}
$$

welches auch die Werthe der $\frac{n}{2}$ Parameter $m_{r}, \mu_{r}$ sein mägen. Daraus folgt, das jede lineare Reilue $\xi_{i}+\zeta$ bei der Tramsformation in sich verschoben wird. Cuter diesen befinden sich insbesondere erzeagende lineare Reilien der Fliche, wenn:

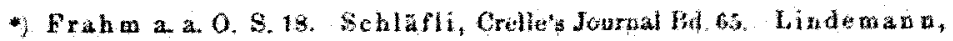
Projectivische Mechanik, Math. Aunales Bd. VII. 


$$
\sum m_{r} \mu_{r}=0
$$

Der Ausdruck $z_{i}+\xi_{i}$ aber stell überhaupt sämmtliche Punkte der Flache vor, denn man kann za jedem Werthsystem $a_{i}=z_{i}+\xi_{i}$ die zugehörigen Parameter $w_{r}, \mu_{r}$ aus Gleichungen mit nicht verschwindender Determinante bestimmen. Demnach hat die Transformation die Eigensellaft, ein System von Erzeugenden der Fläche so in sich zu transformiren, dass jede Erzeugende festbleibt, während ihre Punhte sich verschieben. Jedes derartige System enthält $\infty^{n-3}$ gerade Linien mit $\infty^{n-2}$, a. h. sämmatlichen Flüchempunhten.

Es giebt $2^{\frac{n}{2}-1}$ solcher Systeme, wie schon oben bemerkt wurde. Die Erceugenden ein und desselben Systems schneiden sich nur in solchen Punkten, die den Mannigfaltigkeiten $z_{i}$ oder $\xi_{i}$ angehören.

Man Jann demnach die Fläche durch vertauschbaxe Substitutionen, bei desien je ein System von Erzeugenden fest blcibt, in sich transfor. miren. Da jede Substitution eise wesentliche Constante einführt, so werden mindestens $\frac{n}{z}$ Transformationen dieser Art erforderlich sein, wenn eine gegebene Transformation entstehen soll.

Eine ganz bestimnte Anordnung dieser $\frac{n}{2}$ Trubsformationen entsteht nur für den Fall $n=4$, Sollen die beiden Transformationen:

$$
\begin{array}{ll}
l_{1}^{\prime}=l_{2}{ }^{2}=k_{1} ; & l_{1}{ }^{2}=\lambda_{2}{ }^{2}=k_{2}, \\
\lambda_{1}=\lambda_{2}=\frac{\omega_{1}}{k_{3}}, & l_{2}{ }^{2}=\lambda_{1}{ }^{2}=\frac{\omega_{2}}{l_{2}},
\end{array}
$$

sich zu einer gegebenen mit den $W$ urzeln $r_{1}, \varrho_{1} ; r_{2}, \varrho_{2}$ zusammensetzen, so hat man:

also:

$$
\begin{aligned}
& k_{1} k_{2}=r_{1}, \quad \frac{\omega_{1} \omega_{2}}{k_{1} k_{7}}=\rho_{1}, \\
& k_{1} \omega_{2}=r_{2}, \quad \omega_{1} k_{2}=o_{2},
\end{aligned}
$$

$$
\begin{array}{ll}
k_{1}=\sqrt{r_{1} r_{2},} & \frac{\omega_{1}}{k_{1}} \equiv \sqrt{\rho_{1} \rho_{2}}, \\
k_{2}=\sqrt{\rho_{2} r_{1},} & \frac{\omega_{2}}{k_{2}}=\sqrt{\rho_{1} r_{2}},
\end{array}
$$

womit die gegebene Transformation in zwei vertauschbare zerlegt ist, von denen jede ein System von Erzeugenden ungeändert lässt.

Daggegen hat man für $n=6$ vier Transformationen dieser Art, von denen je drei schon genügen, um eine gegebene mit den Wurceln $r_{1}, \rho_{1} ; r_{2}, \rho_{2} ; r_{3}, \rho_{3}$ hervorzubringen $*$ ).

- Verschieden von dieser Anffasung der Systeme der Erzeugenden einer $F_{z}$ in einem hoheren Roume ist die, weiche Her Cayley gelegentlich entwickelt 
s.

\section{Specielle Substitutionen.}

Eine uneigentliche specielle Sulstitution ist durch die Gleichungen:

$$
y_{i}=x_{i}-2 a_{i} \frac{a_{z}}{\left\langle a^{2}\right\rangle}
$$

gegeben, in denen man noch zar Vereinfachung $\left(a^{2}\right)=\sum a_{i}^{2}=1$ setzen kann. Man kann diese Transformationen auch als Centraltarojection der Fläche $f=0$ vom Punlti $a$ aus in sich selhat bezeichnen. Es sind dies zugleich diejenigen Transformationen, welehe Herr Klein gelegentlich als Spiegelungen bezeichnet hat, wulches Ausdruckes wir uns im Folgenden gleichfalls bedienen.

bat $(O$ n the superlines of a quadric surface in five dirnenvional space, Quarterly Journal, vol. Xlt, p. 176). Wern die Gleiohung der Flache in der Form:

$$
\sum x_{i}^{2}-\sum y_{i}^{2}=0, \quad i=1 \ldots . .
$$

geschrieben wird, so setze man:

$$
y_{i}= \pm a_{i k} x_{k}
$$

Sind die $\alpha_{i k}$ Coefficienten einer orthogonalen Substitution der Determinante \pm 1 mit $\frac{*}{2}$ homogenen Variabelen, so erfillen die Gleichungea (2) offenbar die Bedingung (1). Die Gleichungen (z) repräsentirea ai al Xanuigfiltigkeit, welche für eine eigentliche Substitution als Erocugesule erster $A n t$ in anderen Falle als eine

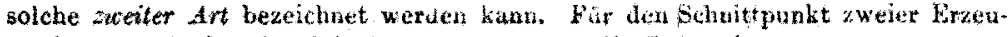
genden (2) mit den Cocffeienten $\alpha_{i k}, \beta_{i k}$ niass die Determinante

$$
a_{1 k}-\beta_{12}
$$

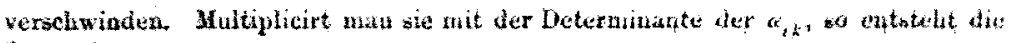
Deterwiniute:

$$
\mid 1-\sum_{j i} \beta_{i k}:
$$

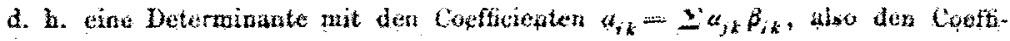
cienten ciner orthogonalex Substitution, welche ilus den isiaten der $\alpha_{k}$ wad $\beta_{i k}$

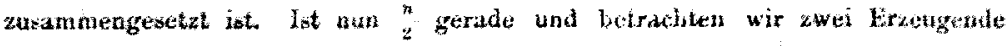
gleicher Art, so sint die $a_{i j}$ Coefficiented ciner eigenthichen Shtwtitution, wn

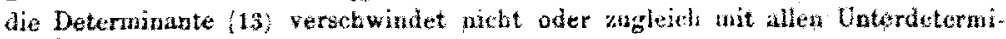
nanten bis zu einer ungeraden Ordnum. Fär Erzeggende angleicher Art ver.

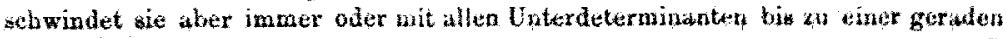

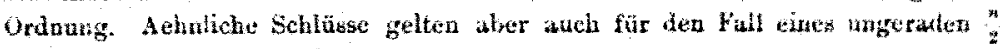
und man bat daher den Satz:

Zwei Erzeugevide gleicher oder angleicher, Ant ahoiden sich in Allgemeinen

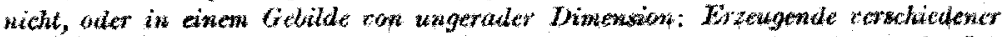

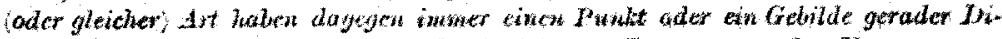
mension gemein, je nathdem die Dimewaion des Hawme ou der Form 4 - 1 (oder +1$)$ ist. 
Jede uneigentliche (eigentliche) Substitution lässt sich aus einer eigentlichen (uneigentlichen) und einer speciellcu der Art (1) zusammensetzen. Denu aus der Verbindung ron (1) mit:

$$
z_{i}=\sum a_{i k} x_{k}
$$

entsteht:

$$
z_{i}=\sum a_{i k}\left(x_{k}-2 a_{k} u_{x}\right)
$$

so dass die Coefficienten der neuen Substitution werden:

$$
b_{i k}=a_{i k}-2 a_{k} \sum a_{i r} a_{r}
$$

Daraus ist umgekehrt:

also:

$$
\sum a_{k} b_{i k}=-\sum a_{k} a_{i k}
$$

$$
a_{i k}=b_{i k}-2 a_{k} \sum b_{i r} a_{r}
$$

woraus, wenn die $b_{i k}$ gegeben sind, bei beliebigem $a_{i}$ die $a_{i k}$ bestimmt werden können. Somit ist die Substitution mit den Coefficienten $b_{i k}$ in $\mathrm{zwei}$ andere zerlegt, von denen die eine die willkürliche uneigentliche der $a_{i}$ ist. Beide sind in Allgemeinen nicht vertauschbar.

Betrachtet mau die $b_{i k}$ als zu einer uneigentlichen Substitution gehörig, so hat man, den Wurzeln $\pm \sqrt{m}$ entsprechenl, die beiden Gleichungen:

$$
\begin{aligned}
& \sum \alpha_{r} b_{i r}=+\sqrt{m \alpha_{i}}, \\
& \sum \beta_{r} b_{i r}=-\sqrt{m \beta_{i}},
\end{aligned}
$$

Verwendet man die $\alpha, \beta$ zur linearen Transformation (1), so hat man nach (5):

$$
\begin{aligned}
& a_{i k}=b_{i k}-2 \alpha_{i} \alpha_{k} \sqrt{m}, \\
& a_{i k}^{\prime}=b_{i k}+2 \beta_{i} \beta_{k} \sqrt{m},
\end{aligned}
$$

und damit zwei eigentliche Transformationen, die respectire mit derjenigen, aus der sie hergeleitet wurden, vertauschbar sind. Endlich bestehen noch die Gleichungen:

$$
\begin{aligned}
& \sum a_{i k} \alpha_{k}=-\alpha_{i} \sqrt{m}, \\
& \sum a_{i k} \beta_{k}=-\beta_{i} \sqrt{m}, \\
& \sum a_{i k}^{\prime} \alpha_{k}=+\alpha_{i} \sqrt{m}, \\
& \sum a_{i k}^{\prime} \beta_{k}=+\beta_{i} \sqrt{m},
\end{aligned}
$$

d. b. die eigentlichen Substitutionen der $a_{i k}, a_{i k}^{\prime}$ sind selbst ron speciellem Charakter, da ihre Determinante $\Omega(\rho)$ die Doppelwurzeln $+\sqrt{m},-\sqrt{m}$ besitzt. Somit hat man den folgenden Satz: 
Jede (allgemeine) uneigentliche Substitution lässt sich auf atwiefache Weise in ein vertatuschbares System zweicr specieller Substitutionen zerlegen, von dener die eine eine Substitution von der Art (1), d. h. eine Centralprojection der Flläche $f$ vonu Punkte $\alpha(\beta)$ aus, dic andere eine Bewegung von speciellem Charahter ist, bei der eine Gerade fest bleibt.

Eine andere Classe besonderer Substitutioneu bilden die symmetrischer, bei denen $a_{i k}=a_{k i}$ ist.

Sie führen, wie bereits früher gezeigt wurde*), auf eine Determinante $\Omega(\rho)$, welche im allgemeinsten Falle nur die Wurzeln $\pm \sqrt{m}$ eine jede $\frac{n}{2}$ mal besitzt, und sind eigentlich oder uneigentlich, je wachdem $n$ durch 4 theilbar ist oder nicht. Die quadratische Form

$$
\sum a_{i k} x_{i} x_{k}=F
$$

ist dann von speciellem Charakter, weil sie der Gleichung:

$$
\sum\left(\begin{array}{c}
\partial \hat{k}^{\prime} \\
\partial x_{i}
\end{array}\right)^{2}=4 m \sum x_{i}^{2}
$$

genügt. Diese Substitutionen spielen eine wesentliche Rolle bei der Untersuchung der quadratischen Formen, welche jener Gleichung genügen**). Bezeichnet man die halben Differentialquotienten von $F$ durch $F_{i}=\frac{1}{2} \frac{\partial F}{\partial x_{i}}$, so lässt sich die symmetrische Substitution in der Form:

$$
y_{i}=F_{i}
$$

schreiben.

Jede (allgemeine) eigentliche Substitution kann auf unendlich viele Arten in die Folge von zwei symmetrischen Substitutionen zerlegt werden.

Um dies zu beweisen, betrachten wir die beidell Formen:

$$
\left\{\begin{array}{l}
F=\sum X_{i}{ }^{2} l_{i}+\Xi_{i}{ }^{2} \lambda_{i}, \\
\Phi=\sum X_{i}{ }^{2} l_{i}+\Xi_{i}{ }^{2} \lambda_{i},
\end{array}\right.
$$

in denen $l_{i} \lambda_{i}=\omega, l_{i}^{\prime} \lambda_{i}^{\prime}=\omega^{\prime}$ sein möge. Beide genägen der Gleichung (6) und vermitteln die beiden symmetrischen Transformationen:

$$
\left\{\begin{array}{l}
y_{i}=F_{i}, \\
y_{i}=\Phi_{i}
\end{array}\right.
$$

welche nach einander ausgeführt werden sollen. Unter geeigneter Wahl der Coefficienten $l, \lambda$ ergiebt sich dadurch die Substitntion der. $a_{i k}$ (\$ IV. (10j). 
Man hat zunächst:

$$
\begin{aligned}
& F_{k}=\sum X_{i} l_{i} x_{k}^{i}+\Xi_{i} \lambda_{i} y_{k}^{i}, \\
& \Phi_{k}=\sum X_{i} l_{i}^{\prime} x_{k}^{i}+\equiv_{i} \dot{\lambda}_{i}^{i} y_{k}^{i},
\end{aligned}
$$

oder bei vollstïndiger Ausführung an Stelle von (9):

$$
\begin{aligned}
& y_{i}=\sum x_{k} x_{k}^{s} l_{s} x_{i}^{s}+\sum x_{k} y_{i}^{*} \lambda_{s} y_{i}^{*}, \\
& y_{i}=\sum x_{m} x_{s}^{t} l_{t}^{\prime} x_{i}^{t}+\sum x_{m} y_{m}^{\prime} \lambda_{i}^{\prime} y_{i}^{t},
\end{aligned}
$$

und durch Zusammensetzung beider Substitutionen:

$$
\begin{aligned}
z_{i} & =\sum x_{m}^{s} x_{i}^{*} l_{t}^{\prime}\left(x_{k} x_{k}^{*} l_{s} x_{m}^{s}+x_{k} x_{j k}^{*} \lambda_{s} y_{m}^{\prime}\right) \\
& +\sum y_{m}^{e} y_{i}^{t} \lambda_{i}^{\prime}\left(x_{k} x_{k}^{s} l_{s} x_{m}^{s}+x_{k} x_{j k}^{*} \lambda_{s} x_{m}^{s}\right)
\end{aligned}
$$

Vereinfacht mali vermittelst $\S I$. (9) die Combinationen der

so ergiebt sich:

$$
\sum x_{k}^{i} y_{k}^{i} \text { etc. }
$$

d. h. eine Transformation mit den Coefficienten $b_{i k}$ :

$$
b_{i k}=\sum y_{k}^{s} x_{i}^{s} l_{s}^{\prime} \lambda_{s}+z_{s}^{\prime} l_{s} y_{i}^{s} x_{k}^{s} \text {. }
$$

Die $b_{i k}$ sind nacb $\$$ IV. (10) identisch mit den $a_{i k}$, wenn:

Daraus folgt:

$$
\lambda_{s}^{\prime} \lambda_{s}=r_{d}, \quad \lambda_{s}^{\prime} l_{s}=\rho_{s}
$$

$$
\text { w) } \frac{l_{s}^{\prime}}{l_{s}^{\prime}}=r_{s}, \quad \omega^{\prime} \frac{l_{s}}{l_{s}^{\prime}}=Q_{s}
$$

oder:

$$
\omega \omega^{\prime}=m, \quad l_{s}^{\prime}=\frac{r_{s} l_{s}}{\omega} .
$$

Die Grössen $l_{s}$ bleiben daher völlig willkürlich, wonach die vorgelegte Transformation auf unendlich vielfache Weise aus zwei symmetrischen (nicht vertauschbaren) zusammengesetzt werden kann. Eine ganz specielle Zerlegung dieser Art ist z. B. durch die beiden Formen:

vermittelt.

$$
\begin{aligned}
& F=\sum x_{i}^{*}+\equiv_{i}^{*}, \\
& \Phi=\sum x_{i} v_{i}+\equiv_{i}^{*} o_{i}
\end{aligned}
$$

Wir wenden uns jetzt wieder zur Betrachtung specieller uneigentlicher Transformationen (1). Zwei Substitutionen:

$$
\left\{\begin{array}{l}
y_{i}=x_{i}-2 a_{i} a_{x} \\
y_{i}=x_{i}-2 b_{i} b_{x}
\end{array}\right.
$$


sind im Allgemeinen nicht vertauschbar, deun combinirt liefern sie die Substitution:

$$
z_{i}=x_{i}-2 a_{i} a_{z}-2 b_{z} b_{i}+4(a b) a_{z} b_{i} .
$$

Die Bedingung der Vortauschlarkeit ist:

$$
\sum\left(a_{i} b_{i}\right)=(a b)=0
$$

d. $h$. die inolutorische Beziehung der Coefficionten $a_{i}, b_{i}$, Zuei solche Transformationen (13) liefern dieselle symmetrische sigentliche Transformation:

$$
z_{i}=x_{i}-2 a_{i} a_{x}-2 b_{x} b_{i},
$$

in welcher Reihenfolge sie auch angewandt werden*).

Setzt man beliebig viele unter einander vertauschbare Transformationen dieser Art, $a, b, c, \cdots$, zusammen, so entsteht die folgende:

$$
z_{i}=x_{i}-2 a_{i} \alpha_{z}-2 b_{x} b_{i}-2 c_{x} c_{i}-\cdots
$$

Je $n$ derselben hinter einapler angewand, lieforn aber tine identische Transformation. Es ergriebt sich dies aus der Identitit:

$$
-x_{i}=x_{i}-2 a_{i} a_{x}-2 b_{i} b_{x}-2 c_{i} c_{7} \cdots
$$

Man erhält dieselbe, wenn man die identisch verschwindende Determinante:

$$
\mid \begin{array}{ccccc}
a_{1} & a_{2} & \cdots & a_{n} & a_{x} \\
b_{1} & b_{2} & \cdots & b_{n} & b_{x} \\
t_{1} & c_{2} & \cdots & c_{n} & c_{x} \\
\vdots & \vdots & & \vdots & \vdots \\
x_{1} & x_{2} & \cdots & x_{n} & v_{x_{2}^{2}}
\end{array}=0
$$

mit der Determinante der $a, b, c, \ldots$ multiplicirt und dio entstehende Identität nach $x_{i}$ differentlirt.

Es lässt sich nachweisen, dass ausser dieser idenhischen Sulstitution, welche durch ne vertauschlore Substitutionen (1) eermittelt wird, laino andere Gruppe on is nistcertauschlaren Transformalumen der Art (1) vorhanden ist, wellhe ebetfalls ane idontische Transfomation liefert.

Betrachtet man nämlich die Transformation (1) als eine Ceptralprojection der Fläche $f=0$ in sich selbst, bei wether je zwei auf einer durch den Punkt a gehenden Geraden gelegene Flächetupunkte mit einander vertanscht werden, so ergight sich leicht: Soll überbaupt eine identische Transformation durch $n$-nalige Cestralprojection entstehen, so muss jede Gruppe von n-w 1 Projectionscentren mit sänmtlichen Berahrungspankten der rom $n^{\text {tea }}$ an die Flache gelegten Tan-

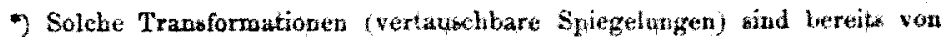
den Geometern mehriach verwerdet. $V_{g}$. 2. B. Lie, Particile lifferentialgleachungen and Complexe, Math. Antalen Bd. V, 1. 180, 182. 
genten in einer Ebene liegen, d, h. je $n-1$ der Punkte $a, b, c$ müissen sich in der Polarebene des $n^{\text {ien }}$ befinden, wodurch gerade die $\mathrm{Be}$ dingungen der Involution $(a b)=0,(a c)=0, \cdots,(b c)=0, \cdots$ berbeigefuhrt werden.

Eine jede symmetrische Transformation lässt sich in $\frac{n}{2}$ specielle uncigentliehe Sulstitutionen zerlegen, die unter sich vertauschbar sind. Un dies zu beweisen, gehe man von der Form symmetrischer Transformationen ans, welche $\frac{n}{2}$ Systeme von Coefficienten $a, b, c, \cdots$ verwendet, und durch die specielle quadratische form:

$$
F=\left|\begin{array}{ccccc}
\left(a^{2}\right) & (a b) & (a c) & \cdots & a_{x} \\
(a b) & \left(b^{2}\right) & (b c) & \cdots & b_{x} \\
(a c) & (c b) & \left(c^{2}\right) & \cdots & c_{x} \\
a_{x} & b_{x} & c_{x} & \cdots & \Sigma_{x_{i}}
\end{array}\right|
$$

vermittelt ist*). Der Differentialquotient $F_{i}$ hat den Werth:

$$
F_{i}=\left|\begin{array}{cccc}
\left(a^{2}\right) & (a b) & \cdots & a_{i} \\
(a b) & \left(b^{2}\right) & \cdots & b_{i} \\
\vdots & \vdots & & \vdots \\
a_{x} & b_{x} & \cdots & b_{2} x_{i}
\end{array}\right|
$$

Multiplicirt man nun $F$ mit dem Quadrat der Determinante:

$$
\left|\begin{array}{cccc}
\lambda_{1} & \lambda_{2} & \cdots & \lambda_{s} \\
\mu_{1} & \mu_{2} & \cdots & \mu_{s} \\
\vdots & \vdots & & \vdots \\
\nu_{s} & \nu_{s} & \cdots & \nu_{s}
\end{array}\right|,
$$

welche $\frac{n}{z}=s$ Reihen besitzt, so kann man an Stelle der $a, b, c, \cdots$ solche lineare Combinationen derselben mit Hülfe der Parameter $\lambda$, $\mu, \cdots, \nu$ einführen, dass in $F_{i}$ alle nicht zur Hauptdiagonale gehörenden Coefficienten fortfallen, womit $F_{i}$ sich in

$$
x_{i}-2 \alpha_{x} \alpha_{i}-2 \beta_{x} \beta_{i}-2 \gamma_{x} \gamma_{i} \ldots
$$

verwandelt und die allgemeine symmetrische Substitution in $\frac{n}{z}$ vertauschbare specielle (1) zerlegt ist.

Man kann damach die allgemeine eigentliche Substitution in zwei symmetrische Transformationen zerlegen, deren jede aus einer Gruppe von $\frac{*}{2}$ unter sich vertauschbaren Spiegelungen besteht.

Wir untersuchen schliesslich die fortgesetate Anwendung uneigentlicher specieller Substitutionen tuberhaupt. In Ganzen verlangt eine

*) Voss, Math Awualen Bd. X, p. 146. 
orthogonale Substitution $\frac{n}{\mathrm{~g}}(n-1)$ willkürtiche Parameler. Da uun jede Substitution (1) schon $n-1$ enthalt, so wärden bereits $s=$ derselben combinirt die hinreichende Allgemeinheit zu besitzen seheinen. Aber man kanu sich leicht iberzeugen, dass selbst die Anwendung von $n-2$ Substitutionen (1) immer noch keine allgemeine eigonlliche Substitution liefert. Fs wird daher eines besondern Beweises bedürfeu, dass eine jede eigentliche Substitution als Folge von $n$ speciellen uneigentlichen Substitationen angesehen werden kaun.

Obwohl von uns schon pben eine Zerlegung der Substitution in $n$ specielle von besonderem Charakter gelehrt ist, so wollen wir doch noch eine Muthode angeben, welche diret die Confficionten $a_{i 1}$ dieser gegebenen eigentlicken Substitution in solche umwandelt, wie sie aus $n$ hinter einander angewandten van der Form (1) entstehen.

Die Coefficienten der orthogonalen Substitution, welche entsteht, wenn man successive specielle Trapsformationen $(1 ; a, b, c, \cdots$ auweudet, besitzen schon bei viermaliger Wiederholung eine sehr unübersichtliche Form. Wir suchen daher zunächst die aus $k$ successiven Transformationen dieser Art eptstehenden Cuefficienten übersichtlicher darzustellen.

Wir bezeichnen diese Cueficienten durch $\alpha_{4 k}$; ihre Determinante ist gleich $(-1)^{k}$. Man hat feruer die Identität:

oder:

$$
\begin{array}{cc:c}
\alpha_{i k} & u_{i} \\
v_{k} & 0
\end{array} \alpha_{i k}=-\sum u_{i} v_{k} \alpha_{i k}
$$

$$
\begin{array}{ll}
\alpha_{i k} & u_{i} \\
u_{k} & 0
\end{array}=(-1)^{k-1} \sum u_{i} v_{k} \alpha_{i k}
$$

d. h. man kann die Coefficienten $a_{i k}$ als ihren eigenen eraten Unter. determinauten proportional ansehep*). Macht man you dieser Dar. stellung Gebrauch, so erhält man ohne besondere Schwierigkeit durch geeignete Determinantenreductionen als Werth des Ausdruckes rechter Hand is (19) die übersichtlichere Determinante:

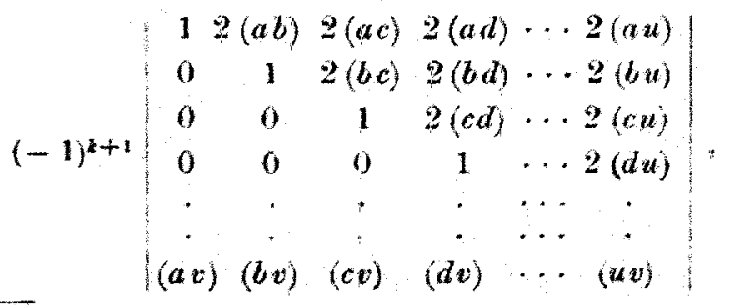

Daltzer, Det. p. 173. 
Sie liefert das Bildungsgesetz der $\alpha_{i k}$, denn $\alpha_{i k}$ ist der Coefficient son $u_{i} v_{k}$ in der Determinante (20). Bezeichnen wir die Determinante, welche durch Weglassung des letaten Elementes $(u v)$ entsteht, durch $\Delta$, so hat man:

$$
\sum u_{i} v_{k} \alpha_{i k}=\Delta^{\prime}+(u v) \text {. }
$$

Wir wollen jetat $L=n$ (gerade) voraussetzen und eine eigentliche Substitution betrachten, welche durch die Cayley'schen Formeln:

$$
a_{i k}=2 \beta_{i k} \frac{\omega}{\mathrm{B}}-[i i]
$$

gegeben ist; die $\boldsymbol{\beta}_{i k}$ sind die Unterdeterminanten der schiefen Determinante, der $b_{i k}, \mathrm{~B}(\S \mathrm{II}$.$) , und [i k]$ nur dann ron Null verschieden and gleich eins, wenn $i=k$. Daher ist:

$$
\sum a_{i k} u_{i} v_{k}=\frac{2 \omega}{B} \sum \beta_{i k} u_{i} v_{k}-(u v) .
$$
diugangen:

Unterwirft man die Coefficienten $a, b, c$ jetat den $\frac{n}{2}(n-1)$ Be(24) $\left\{\begin{array}{cc}\sum b_{i r} a_{i} b_{r}=0, & \sum b_{i r} b_{i} c_{r}=0, \\ \sum b_{r i} a_{i} c_{r}=0, & \sum b_{i r} b_{i} a_{r}=0 \\ \sum b_{r i} a_{i} a_{r}=0, & \vdots \\ \vdots & \end{array}\right.$ welche wegen $b_{i k}=-b_{k i}, b_{i i}=b_{k i}=\omega$ die weiteren Relationen:

$$
\left\{\begin{array}{c}
\sum b_{r i} a_{i} a_{r}=\omega \\
\sum b_{r i} b_{i} b_{r}=\omega \\
\sum b_{r i} c_{i} c_{r}=\omega \\
:
\end{array}\right.
$$

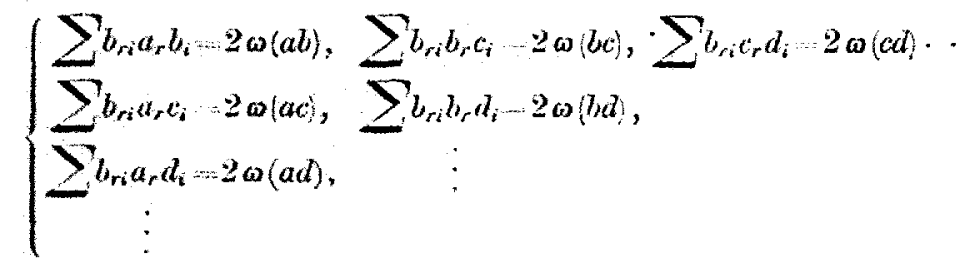

wach sich ciehen, so lässt sich zeigren, dass aus den den Gleichungen (24) onterworfenen Coefficienten von $n$ speciellen uneigentlichen Trausformationen gerade die vorgelegte der Coefficienten $a_{i k}$ hervorgeht. 
Zu diesem $Z$ wecke bezeichnen wir die Deterninante der $a, b, c$, ... durch $\Delta^{\prime \prime}$. Dann ergiebt sich:

$$
\begin{aligned}
& b_{11} b_{12} \cdots b_{1 *} u_{1} \\
& b_{y_{1}} \quad b_{y} \ldots b_{y_{*}} \text { at }
\end{aligned}
$$

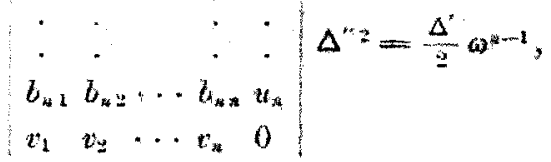

nach welcher Gleichung:

$$
\sum u_{i} v_{k} \beta_{i k}=-\frac{1}{ \pm \Delta^{*}} \omega^{n-3},
$$

oder nach $(21),(23)$ :

$$
\begin{aligned}
& \sum u_{i} v_{k} \beta_{i k}=\mathrm{B}\left[\sum u_{i} v_{1} a_{i k}+(u v)\right] \\
& \Delta^{\prime}=\sum\left[u_{i} a_{i} a_{i k}-\left(u_{i}\right)\right],
\end{aligned}
$$

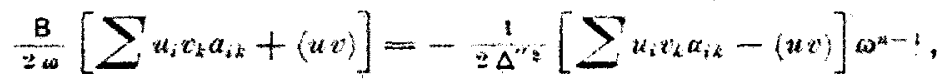

und endlich wegen:

$$
\begin{aligned}
& B \Delta^{\prime \prime 2}=\omega^{n} \\
& a_{i k}=-\alpha_{i k} .
\end{aligned}
$$

Damit sind die $a_{i k}$ in eine form gebracht, in welcher sie direct als Coefficienten der $n$ mal wiederholten unelgentlichea Trapsformation erscheinen. Die $a_{i}$ sind dabei noch ganz willkürich, die $l_{i}$ einer, die $c_{i}$ wei linearen Gleichungen unterworfen, and so fort. Man kan doher Wurch eine Construction, welche der eines I'turedies einer Fläh zoeiten Grades ganz analog rerläuft, beliebig wiek solker Gruppen won

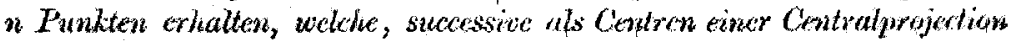
benutzl, die gegebene Transformation hercorloringen.

Liegt eine uneigentliche Substitution vor, so kann man sie zuaüchst in eine specielle uneigentliche und eine eigentliche mit der Doppelwurzel 1 zerlegen. Die letztere lisst sich dann jedenfalls ans $n-2$ uneigentlichen spreiellen in der vorhin beschriebenen Weise an. sammensetzen, so dass im Garam eine uneigmaliche Sulstitatiom auf

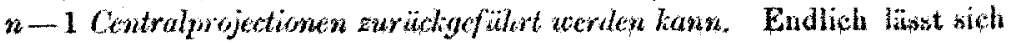
leicht erkemen, dass sich bei ungeradem $n$ schon $n-1$ Transformatiouen von der Art (1) zu einer gegebenen zusammensetzen lassen.

Wir schliessen hieran noch folgende Bemerkung. Die Cayley'sehen Formeln setzen uns in den Stand, eine allgeweine eigentliche Substitution mit der Determinante +1 hinzuschreiben. Es spll hiter noch gezeigt werden, wic man sich dersoltuen bedienen kann, um 
eigentliche Substitutionen auzageben, deren Determinante $\Omega(\rho)$ die Doppelwurzel +1 oder -1 oder beide gleichzeitig besitat.

Nimmt man eine allgemeine uneigentliche Substitution:

$$
z_{i}=\sum b_{i k} x_{k}
$$

so entsteht daraus darch Anwendung der speciellen

die eigentliche

$$
y_{i}=z_{i}-2 t_{i} b_{i}
$$

$$
y_{i}=\sum a_{i k} x_{k}, \quad a_{i k}=b_{i k}-2 b_{i} \sum b_{r} b_{r k} .
$$

Demnach ist die Determinante $\Omega(\varrho)$ gleich:

$$
\left|\begin{array}{cc}
b_{i k}-\varrho & b_{i} \\
2 \varrho b_{k} & -1
\end{array}\right|
$$

Soll nun $\Omega(1)=0$ sein, so hat man als einzige Bedingung:

$$
(a b)=0 \text {, }
$$

zu yelcher noch die analoge:

$$
(\beta b)=0
$$

hinzukonmt, wemu auch $\Omega(-1)$ verschwinden soll. Die $\alpha, \beta$ haben labei die unter (5) p. 342 angegebene Bedeutung.

$$
\text { \$ VI. }
$$

\section{Besondere Fälle.}

Wir werden jetzt die Falle $n=2,3,4,6$ der orthogonalen Substitutionen besprechen, welche voraugsweise in geometrischen Untersuchungen Verwendung finden.

A. Es sei $n=2$; Substitution im binären Getret.

Die allgemeing uneigentlicle Substitution ist zugleich nach $\$$. die specielle. Dagegen nuss die eigentliche Substitution aus zwei speciellen uneigentlichen (Spiegelungen) zusammengesetzt werden. Nimmt man wach Baltzer*) als Coefficienten der gegebenen Substitution die folgenden:

$$
\begin{aligned}
& a_{11}=\frac{1-\lambda^{2}}{1+\lambda^{2}}, \\
& a_{21}=\frac{-2 \lambda}{1+\lambda^{2}},
\end{aligned} \quad a_{22}=\frac{2 z}{1+\lambda^{2}}, \quad \begin{aligned}
& 1-\lambda^{2} \\
& 1+\lambda^{2}
\end{aligned}, \quad \mathrm{~B}=\left|\begin{array}{cc}
1 & \lambda \\
-\lambda & 1
\end{array}\right|,
$$

so hat man für

oder:

$$
\lambda=\frac{(a b)}{V_{1}-(a b)^{2}},
$$

$$
\lambda=\frac{V \mathbf{i}-(a b)^{2}}{(a b)},
$$


wo:

so ist:

$$
\left(a b_{1}\right)=a_{1} b_{1}+a_{3} b_{4}
$$

$$
\begin{aligned}
& -a_{11}=1-2 a_{1}^{2}-2 b_{1}^{2}+4(a b) a_{1} b_{1}, \\
& -a_{12}=-2 a_{1} a_{2}-2 b_{1} b_{2}+4(a b) a_{2} b_{1} \\
& -a_{21}=-2 a_{1} a_{2}-2 b_{1} b_{2}+4(a b) a_{1} b_{2}, \\
& -a_{22}=1-2 a_{2}^{2}-2 b_{2}^{2}+4(a b) a_{2} b_{2} .
\end{aligned}
$$

Rechterhand stehen jetzt die Coeffeienten, welche durch successive Ausführung der Substitutionen $a, b$ entstehen. Die symmetrische Sub. stitution ist hier zugleich die identische, $(a b)=0$.

In Verbindung mit der am Schluss des $\$$ IV, betrachteten Verlegung einer eigentlichen Substitution in eine Reihe vertauschbaror, deren jede eine $\infty^{-3}$ Seliaar linearer Reihen so ungeandert lässt, diss jede einzelne Reihe nur in sich verschoben wird, welcher letztere Pro. cess auf 2 successiven Spiegelungen beruht, hat man den folgrenden Satn*;:

Fine jede eigentliche Sulstitution in fianme wo $2 k-s$ Dimmsio-

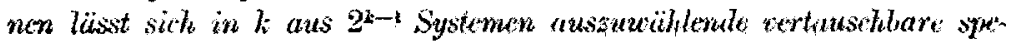
riellere Transformationen zerlegen, dexen jede cin System won Frreagenden viner Flähe zxeiten Grades ungeändert lidsit tond auf der Folyr von zwei spitgelangen berult.

B. Es sei $n=3$; orthoyonale Substitution in ar Ebene.

Die allgemeine Substitution mit positiver Determinante +1 ent. steht durch Verbiudnug aweier uneigentlicher specieller. Setat man nach Baltzer:

$$
\mathrm{B}=\left[\begin{array}{ccc}
1 & \nu & -u \\
-v & 1 & 2 \\
\mu & -\lambda & 1
\end{array}\right)=1+\lambda^{2}+\mu^{2}+y^{2}
$$

so sind die Coefficienten derselben:

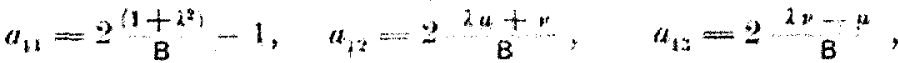

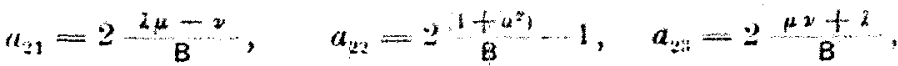

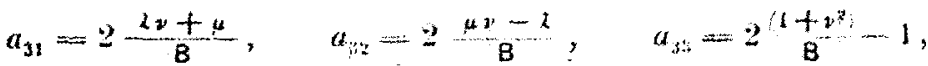

Setzt man:

$$
\begin{gathered}
\lambda(a b)=a_{2} b_{3}-a_{3} b_{2}, \\
\mu(a b)=a_{3} b_{1}-a_{1} b_{3}, \\
\nu(a b)=a_{1} b_{2}-a_{2} b_{3}, \\
\mathrm{~B}=\frac{1}{\left(a b^{2}\right.}=\left[a_{1} b_{1}+a_{2} b_{2}+a_{3} b_{3}\right)^{2},
\end{gathered}
$$

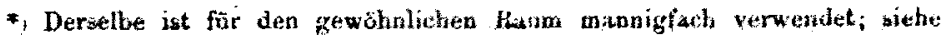

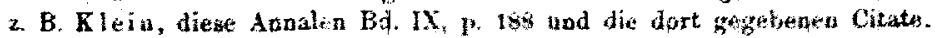


so ergeben sich die Coefficienten $a_{i z}$ in derjenigen Form, welche sie durch die Verbindung der Centralprojectionen des Kegelschnittes $x_{\mathfrak{q}}{ }^{2}+x_{2}{ }^{2}+x_{3}{ }^{2}=0$ von den Punkten $a, b$ aus erhalten würden.

Die genannten Punkte sind auf einer durch den Punkt $\lambda, \mu, \nu$ gehenden Geraden so zu wählen, dass:

$$
B(a b)^{2}=1 \text { oder }=\left(a^{2}\right)\left(b^{2}\right)
$$

je nachdem zwischen den $a_{i}, b_{i}$ die Identität $\left(a^{2}\right)=1,\left(b^{2}\right)=1$ vorausgesetat wird oder nicht.

Zu jedem witliürlich auf dieser Goraden angenommenen Punkte a gehören mithin noch zuei verschiedene $b$, welche dieselbe gegebene Substitution mit a verbunden hervorlringen. Man kann dies auch so ausdrücken:

Coustruirt man in einen Kegelschnitt Vierseite, deren zwei Gegenseiten sich in einem festen Punkte $a$ schneiden, währeud die dritte Seite durch einen festen Punkt $b$ geht, so geht auch die vierte durch einen festen Punkt, weleher mit $a, b$ auf einer Geraden und $z \mathbf{u} b$ in Bezugr auf den Kegelschnitt conjugirt liegt.

Die identische Transformation besteht in der dreimaligen Projection des Kegelsolwittes von den Ecken eines Polardreiechs aus: In einen Kegelschnitt lassen sich unendlich viele Dreiecke beschreiben, deren Seiten durch die Ecken des nämlichen Polardreieckes gehen.

Die Determinante $\Omega(\rho)$ hat im allgemeinen Falle drei verschiedete Wurzeln, also die Charakteristik:

Die besonderen Falle:

$$
[1,1,1] \text {. }
$$

entstehen, je nachdem die Punkte $a, b$ harmouisch conjugirt sind, oder ihre Verbindungslinie den Kegelschnitt berührt.

C. $n=4$. Wir haben dann die Trarsfarmation ciner Fläche zweiten Grades in sich selbst.

Die eigentliche Transformation kann auf uuendlich viele Arten mach $\$$ V. aus vier Centralprojectionen zusammengesetzt werden. Die viermalige Centralprojection von den Ecken eines Polartetraeders in ganz beliebiger Reihenfolge ist eine identische: es giebt $\infty^{2}$ Vierseite, welche in die Fläche so einbeschrieben werden kömnen, dass die Seiten durch die Ecken eines festen Polartetraeders gehen.

[Anmerkung. Betrachtet man also ein Büschel von Flächen mit gemeinsamem Polartetraeder, so lassen sich 8 verschiedene collineare Transformationen angeben, welche jede Fläche, also auch die ihnen geneinsame Rowmmurp vierter Ordnang ungeändert lassen, aus 
welcher Bemerkung man leich eine Reihe bekannter Sätze aber in eine ebene Curve dritter Ofdnung eingeschriebene Vierseite, suwie aber solche, die einer $R_{4}$ eingeschrieben sind, herleitet.]

Andererseits kann die eigentliche Substitution in zwei successive (eigentliche) symmetrische zerlect werden. Die symmetrische Transformation setzt an Stelle eines Punktes der gegebenen Flache den Berührungspunkt einer Ebene, welche dem ersten in Bezug auf eino Fläche polar conjugirt ist, die mit der gegebeneu ein Vierseit von Erzengenden gemein hat. Die symmetrische Traustormation kann noch auf $\infty^{\prime}$ Arten in 2 wei vertususchbare Centralprojectionen zerlegt werden.

Bei der eigentlichen Substitution bleiben ferner in Allgemeginen vier Purkte fest, die Eckpunkte eines von Erzengenden gebildeten Vierseits, in Bezug auf welches als Coordinatentetraeder die Gleichung der Fläche dann $X_{1} \equiv_{1}+X_{2} \equiv_{2}=0$ wird: gleicheteitig geluen alle Flächen des Büschels $X_{1} \Xi_{1}+\lambda X_{2} \Xi_{2}=0$ in sich über, und jede Gerade des Raumes formt sich in eine Tamgente desselben Fliebenoures ans jenem Büschel um, dem sie ursprünglich angehört.

Die eigentlichen Transformationen zerfallen in folgende Classen:

1) $[1,1,1,1]$, der allgemeine Fall,

2) $[2,2], 2$ nicht ausgezeichnete Doppelwurzeln,

3) [(11), $(11]$, desgleichen, mit verschwindenden ersten Unterdeterminanten, wobei alle Erzeugenden eined Systems fest bleiben,

4) [(11), 1, 1], q beliebige Centralprojectionem*) von deu Punkten $a, b$ aus;

5) [11) (11]], Fall, wo a za $b$ conjugirt ist;

6) [31]; zwischen den Courdiuaten von $a, b$ besteht die Gleichung:

$$
(b a)^{2}=\left(a^{2}\right)\left(b^{2}\right)
$$

Bei einer uneigentlichen Sulstitution gehen dugegen alls Fliudwen des Netzes:

$$
\lambda X_{1}^{2}+\mu \equiv_{1}^{2}+2 \nu X_{2} \equiv_{2}=0
$$

in sich äbcr. Diese Flächen berihren die gergebence

$$
X_{1}{ }^{2}+\equiv_{1}^{2}+2 X_{2} \equiv_{i}=0
$$

in zwei verschiedenen Puakten und gehen durch zwei conjugirte Pole derselben; je zwei derselben schueiden sich in Kegelschnitten, welche durch die Substitution in cinasder gegenseltig uhergefubrt werden. Unter diesen Flächen sind besonders berworahebea:

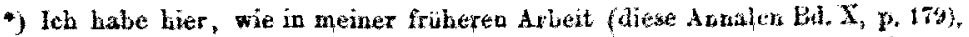

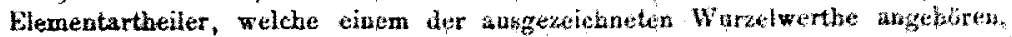
durch einen übergesetzen Strich lisworgenclota. 


$$
\begin{array}{r}
-X_{1}^{2}+\Xi_{1}^{2}+2 X_{2} \equiv_{2}=0, \\
X_{1}^{2}-\Xi_{1}^{2}+2 X_{2} \equiv_{2}=0,
\end{array}
$$

welche mit der gegebenen je eine Berährung längs der Ebenen $X_{1}=0$, $\Xi_{1}=0$ eingehen: die Polarebenen beider Flächen in Bezag auf die Punkte der gegebenen umbüllen diese letztere selbst.

Anmerkung. Man kann sich leicht auch direct von diesen Eigenschaften uneigentlicher Substitutionen überzeugen. $\mathrm{Da}$ bei denselben zwei Punkte auf der Fläche fest bleiben, die beiden anderen Punkte, in denen sich die zugehörigen Erzeugenden schneiden, gegenseitig sich vertauschen, so genügt es, die Fläche $x_{1} x_{2}+x_{3} x_{1}=0$ durch die Gleichungen:

$$
x_{1}=\lambda y_{2}, \quad x_{2}=\mu y_{1}, \quad x_{3}=\nu y_{3}, \quad x_{4}=\rho y_{4}, \quad \lambda \mu=\nu \rho
$$

in sich überzuführen, wobei denn in der That jede Fläche des Netzes:

in sich ubergeht $*$.]

$$
\alpha x_{1} x_{2}+\beta x_{3} x_{4}+\gamma\left[\frac{x_{1}^{2}}{2}+\frac{x_{2}^{2}}{\mu}\right]=0
$$

Die Classification der uneigentlichen Substitutionen ist sehr einfach, da immer die $W$ urzeln $+1,-1$ mit anftreten müssen. Man hat daher die folgenden Fälle:

1) $[1,1,1,1]$, der allgemeine mit zwei reciproken einfachen Wurzeln,

2) $\cdot[3,1]$,

3) $\left[\left(\begin{array}{ll}1 & 1\end{array}\right), 1\right]$ mit einer dreifachen Wurzel +1 , für welche noch alle zweiten Unterdeterminanten von $\Omega(\rho)$ verschwinden (specielle Substitutionen).

D. $n=6$. Wir erhalten die Transformation des Linienraumes in sich, wenn wir die sechs homogenen Variabelen $x$ als Liniencoordinaten in Raume auffassen. Diese Transformationen zerfallen in collineare und reciproke des gewöhnlichen Punkt-Ebenenraumes, je nachdem die Substitution eine eigentliche oder uneigentliche ist**).

Die specielle uneigentlictie Substitution ist diejenige reciproke $\mathrm{Om}$ formung des Punktraumes, welche mit dem linearen Complexe verhnïpft ist, vermöge der an Stelle jedes Punktes die durch ihn gehende Complexebene, an Stelle jeder Geraden ihre reciproke Polare in Bezug auf den Complex tritt. Die identisele Substitution ist vermittelt durch die Umformung in Bezuy auf sechs gegenseitig in Involution liegende lineare Complexe. Die symmetrische allgemeinste Transforwation setat an Stelle

*) VgL insbesondere noch \& VIII.

*) Dieser widhtige Satz rührt von Herrn Klein her, vgl. Math. Annalen Bd. IV, S. 356 . 
irgend einer Geraden ihre reciproke Polare in Besug auf eine betiudiye Fläche sweiten Grades*); ausser dieser uneigentlicher Substitution existirt noch eine symmetrische eigentliche, es ist die (collinearc) $\mathrm{Cm}_{\mathrm{m}}$. formung in Bezug auf zuoci in Involution liegende linearc (onuplexe**).

Wir betrachten zunächst die collineare Sutustitution. Im Allgemeinen bleiben sechs specielle lineare Complexe fest, deren . Axen die Kanten eines Tetracders lillen. Die Gesammtheit der Complexe zweiten Grades, welche in sich äbergehen, wird durch die Gruppe quadratischer Fornen:

$$
\lambda X_{1} \equiv_{1}+\mu X_{2} \equiv_{2}+\nu X_{3} \equiv_{3}=0
$$

gebildet, welche wegen der Identität:

$$
x_{1} \equiv_{1}+X_{3} \equiv_{2}+X_{3} \equiv_{3}=0
$$

ein Büschel tetraedraler Complexe darstellen.

Andererseits geht aus unseren früheren Ueberlegungen hervor, dass die Collineation sich auf untendlich vielo Arten in acei successibe Polurreciprwituten zerleyen lässt in Bezug auf Flächen, welkhe jencs foste Tetraeder zum gemeinsumen Polartetraeder haben. Ebenso kaun sie immer durch Unformungen in Bezug auf sechs lineare Complexe ersetzt werden.

Nach den in $\$$ II. entwickelten Sätzen ist es ferner lejebt, die überhaupt möglichen Substitutionen, nach den Elementartheilern der Determinante $\Omega(e)$ geordnet, anzugeben. Wir füliren dies hier nicht weiter aus, weil bei der Untersuchung über gemeinsame Polartetraeder zweier Flächen zweiten Grades für dis eigentliche Transformation bereits alle Falle aufgexïhlt sind, Die dort aufgestellte 'lisbelle**) gewinnt dadurch noch eine etwas allgemeinere Bedentung. Aber es muss hier bemerkt werden, dass dort zwei lälle, es sind [211), 11], [(211) (11], Nr. 15 und 16, irrthümlich als möglich bezeichnet sind, welche nach den in $\$$ II. bewiesenep allgemeinen Determinanteneigenschaften nicht vorlanden sein können.

Die Eigenschaften der reciproten Sulstitution sind wesextlich andere. Es bleiten im Allgeneing zoci allgeneine lineare Complexe und vier speciulle fost, welche letzteren die Kanten eines Tetractors bilden, de feldenden beiden Kanten sertauschien sich gegensitig.

* Voss, Math. Annalen Bd. X, S. 166.

*) Ebenda S. 16,5 Anm. Diege Tranoformation int nicht wereptlich ver" schicden ron derjenigen, welche an Stelle einer Geraden $x$ fle vierte harmonischic zu zwei fexten Geraden $a, b$ und $x$ anf der Regelactaur $a, b, z$ betat, teren Chasrakteristik [(1111), (11)] ist Tritt an stelle des harmonischen cin belikigh Doppelverbaltris ein, so ist die letzterc $(11111), 1,1$.

**) Vosa, Math. Amalen Bd. X, S. $172 \mathrm{fr}$. 
In sich transformirt werden alle Formen:

$$
\lambda_{1} X_{1}^{2}+\lambda_{2} \equiv_{1}^{2}+2 \lambda_{3} X_{2} \equiv_{2}+2 \lambda_{1} X_{3} \equiv_{3} \text {, }
$$

welche wegen der Identität, welche für alle Gerade des Raumes besteht:

$$
X_{1}{ }^{2}+\equiv_{1}{ }^{2}+2 X_{2} \equiv_{2}+2 X_{3} \equiv_{3}=0
$$

ein Netz quadratischer Complexe bilden. Die Charakteristik derselben:

$$
\left[\begin{array}{llll|ll}
1 & 1 & (1 & 1 & (1 & 1
\end{array}\right]
$$

dentet nach Herrn W eiler's Arbeit*) auf eine Schaar von Complexen, von denen je einfach unerallich viele die nämliche aus zwei Flächen zweiten Grades bestehende singuläre Fläche besitzen.

Diese Flïchen zweiten Grades gehen, wie man leicht sieht, gegentseitig in einander über. In der That entriehmen wir aus \$ III. direct die Gleichung der Schaar specieller Complexe zweiten Grades, welche gegenseitig in sich übergehen:

$$
\sqrt{1-\lambda^{2}} X_{1}^{2}-\sqrt{1}-\lambda^{2} \equiv_{1}^{2} \pm 2 \lambda X_{1} \equiv_{1}+2 X_{2} \equiv_{2}-2 X_{3} \equiv_{3}=0 \text {, }
$$

welche mit Hülfe der Identitat auch in die Form:

$$
\left[\wedge X_{1} \pm \sqrt{1-\Lambda^{2}} \equiv_{1}\right]^{2}+2 X_{2} \equiv_{2}=0
$$

gebracht werden kann. Und diese Schaar stellt zugleich die sämmtlichen singulären Flächen vor, die in dem angegebenen Netze vorhanden sind. Wir schreiben die Gleichung derselben der bequemeren Untersuchung halber in der Gestalt:

$$
\lambda_{1} x_{2}{ }^{2}+\lambda_{2} x_{2}{ }^{2}+\lambda_{3}\left(x_{3}{ }^{2}+x_{4}{ }^{2}\right)+\lambda_{1}\left[x_{3}{ }^{2}+x_{6}{ }^{2}\right]=0,
$$

wo an Stelle von $X_{1}, \equiv_{1}, 2 X_{2} \equiv_{2}, 2 X_{3} \equiv_{3}$ wieder $x_{1}, x_{2}, x_{3}^{2}+x_{4}^{2}$, $x_{3}{ }^{2}+x_{6}{ }^{2}$, gesetzt ist, damit die Identitat wieder in der form:

$$
x_{1}^{2}+x_{2}^{2}+x_{3}^{2}+x_{4}^{2}+x_{5}^{2}+x_{6}^{2}=0
$$

erscheint. An Stelle von (2) kann man auch nehmen:

$$
\left(\lambda_{1}-\lambda_{1}\right) x_{1}{ }^{2}+\left(\lambda_{2}-\lambda_{1}\right) x_{2}{ }^{2}+\left(\lambda_{3}-\lambda_{4}\right)\left(x_{3}{ }^{2}+x_{4}{ }^{2}\right)=0 \text {, }
$$

oder, wenn:

$$
x_{3}{ }^{2}+x_{4}^{2}=z^{2}, \lambda_{1}-\lambda_{4}, \lambda_{2}-\lambda_{4}, \lambda_{3}-\lambda_{4} \text { gleich } \mu_{1}, \mu_{2}, \mu_{3}
$$

gesetzt werden:

$$
\mu_{1} x_{1}^{2}+\mu_{2} x_{2}^{2}+\mu_{3} z^{2}=0
$$

während die Identität in

abergeht.

$$
x_{1}^{2}+x_{2}^{2}+x_{5}^{2}+x_{6}^{2}+z^{2}=0
$$

Die singulare Fläche von (4) in Liniencoordinaten $y$ ist nun die Discriminante der in $\lambda$ quadratisehen Gleichung:

*) Weiler, Muth. Amnalen Bd. VII, S. 160. 


$$
\frac{\mu_{1} y_{1}^{2}}{1+\lambda \mu_{1}}+\frac{\mu_{2} y_{2}^{2}}{1+1 \mu_{2}}+\mu_{3} \frac{\left(y_{3}^{2}+y_{1}^{2}\right)}{1+\mu_{2}}=0
$$

Diese Discriminante zerfallt aber in die Factoren:

$$
\left\{\begin{array}{l}
\mu_{1} \alpha y_{1}{ }^{2}+\mu_{2} \beta y_{2}{ }^{2}+\mu_{3} \gamma\left(y_{3}{ }^{2}+y_{1}{ }^{2}\right)-2 \sqrt{\mu_{1} \mu_{2} \alpha \beta y_{1} y_{2}=0} \\
\mu_{1} \alpha y_{1}{ }^{2}+\mu_{2} \beta y_{2}{ }^{2}+\mu_{3} \gamma\left(y_{3}{ }^{2}+y_{4}{ }^{2}\right)+2 \sqrt{\mu_{1} \mu_{2} \alpha \beta} y_{1} y_{2}=0
\end{array}\right.
$$
wo:

$$
\begin{aligned}
& \alpha=\mu_{2}-\mu_{3}=\lambda_{2}-\lambda_{3}, \\
& \beta=\mu_{3}-\mu_{1}=\lambda_{3}-\lambda_{1}, \\
& \gamma=\mu_{1}-\mu_{2}=\lambda_{2}-\lambda_{1} .
\end{aligned}
$$

Die Gleichungen (j) sind die von $z$ wei flichen zweiten Grades. Man bann sie durch die eincige Glejchung:

$$
\left.\frac{\left[x_{1} V\left(\lambda_{1}-\lambda_{2}\right)\left(\lambda_{2}-\lambda_{3}\right)\right.}{\lambda_{2}-\lambda_{2} \lambda_{3}-\lambda_{4}} V\left(\lambda_{2}-\lambda_{4}\right)\left(\lambda_{3}-\lambda_{1}\right)\right]^{2}+2 x_{2} \equiv_{2}=0
$$

darstellen, oder auch, weil die Sumre der Guadrate der Coefficienten von $X_{1}, \equiv_{1}$ gleich eins ist, in der oben angeggebenen form:

$$
\left[X_{1} \Lambda+\equiv_{1} \sqrt{1-\Lambda^{2}}+2 X_{2} \equiv_{2}=0\right. \text {. }
$$

Aber es befinden sich unter den Complexen (2) nogh solche, für welche die beiden Gleichungen (b) zusammenfallen, nämlich wenu $\approx$ oder $\beta$ verschwindet. Set $u$ man demgemäss $\lambda_{3}=\lambda_{3}$ oder $\lambda_{1}=\lambda_{3}$, so entstehen zwei besondere Flachen zweiten Grades:

$$
\begin{aligned}
& X_{1}^{2}+2 X_{2} \equiv_{2}=0, \\
& \Xi_{1}^{2}+2 X_{2} \equiv_{2}=0,
\end{aligned}
$$

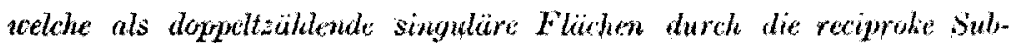
stitution in sich sellst übrothen, wire freilich alus \$ III. direct estnommen werden konnte.

Die Classification der uneigentlichen Substitutionen wird durch den Umstand beträchtlich erleichtert dass iu der Gleichungr $\Omega(0)=0$ immer zwei Worzeln gleich +1 oder -1 siud, und dass jede derselben auch bei höherer Vielfachheit nur in ungerader Anzahl vorkommen kam. Ausserdem hommen die Gesetze des $\$$ II. in Anwendung. Wir habon daher folgende Falle:

1) $[1,1,1,1,1,1]$; der allgemeine, die beiden ersten Elementartheiler beziehen sich auf die Wurceln +1 , die anderen auf zwei Patret reciproker Wurcelu.

2) $[1,1,2,2]$, zwei gleiche Paare reciproker Wurkeln.

3) $[1,1(11)(11)$, desgleichen, mit verschwindenden ersten Vitstdeterminanten.

4) $[3,1,1,1]$, drei Wurceln sind gleich +1 , ausserdem ist ein Paar reciproker vorhanden 
5) $\left[\begin{array}{ll}5 & 1\end{array}\right]$.

6) $[33]$.

7) $[(111\rangle, 1,1,1]$, dreimal wiederhalte uneigentliche specielle Substitation $a, b, c$.

8) $[(111), 3]$, desgleichen, mit einer invarianten Beziehung zwi-

9) $[(311), 1]\}$ schen den $a, b, c$.

10) $\left[\left(\begin{array}{lll}1 & 1 & 1\end{array}\right)(111)\right]$, symmetrische Transformation.

11) $[(11111), 1]$, uneigentliche specielle Substitution.

Die Substitution $[(221), 1]$ ist wahrscheinlich nicht vorhanden. Debrigens würden sich aus 4), 5), 7), 8), 9), 11) noch ebenso viele nicht eigentlich verschiedene Substitutionen ergeben, in welchen die Wurzel - 1 die ausgezejchnete Eigenschaft besitzt, welche in jenen der Wurzel +1 beigelegt worden ist. Sieht man ron diesen $a b$, so kommen za den früher angegebenen 21 eigentlichen Transformationen $*$ ) noch 11 uneigentliche hinzu, so dass im Ganzen 32 Transformationen des Linienrauswes vorkanden sind, welche wesentlich durch die Beschaffenheit der Gebilde sich unterscheiden, welcke bei denselben ungeündert bleiben**).

\section{\& VII.}

\section{Zar Theorie der Reciprocität.}

Die Betrachtungen des vorigen $\$$ haben einige vielleicht bisher nicht bemerhte Eigenschaften der reciproken Punkt-Ebenentransformation ergeben ${ }^{* * *}$ ). Es wird daher nicht urzweckmässig sein, wenn dieselben jetzt direct als eine Folge der reciproken Beziehung nachgewiesen werden.

Auch hier werden wir eine unbeschränkte Zahl von Variabelen voraussetzen. Wir betrachten die Substitution:

$$
x_{i}=\sum a_{i r} x_{r}
$$

in welcher die $n$ Variabeleu $u_{i}$ homogene Ebenencoordinaten, die $x_{i}$ homogene Punktcoordinaten bedeuten. Die Umkehrung der Gleichungen (1) liefert:

$$
u_{i} \Delta_{i s}=\Delta x_{s},
$$

*) Die identische Transfornation ist hier mitgezählt.

**) Trotz vielfacher Versuche babe ich wenigstens das Vorbandensein von weiteren Substitutionen, welche nach § II. denkbar wären, nicht constatiren können.

**) Es ist schon im vorigen $\$$ vielfaltig hervorgetreten, dass in Gebieten von ungeradar Dimension die uneigentliche orthogonale Substitution einen speciellen Charakter gegenüber der eigentlichen besitzt. In ähnlicher Weise verbālt sich die Reciprocität zur Collineation; sehon die Reciprocität der Ebene führt eia gunzes Büschel ron Kegelsctnitten in sich niber. 
wo $\Delta, \Delta_{i s}$ die Determinante und Unterdeterminanten des Systems der $a_{i x}$ bedeuten. Soll die Ebene $u$ den Punkt $x$, dem sie qugeardnet ist, enthalten, so mass:

$$
F=\sum a_{i r} x_{i} x_{r}=0
$$

sein. Die Transformation ordnet aber gleichzeitig jeder Ebene $v$ auch einen Punkt $y$ zu. Setzt man nämlich:

$$
\sum v x=\sum u y,
$$

so hat man, wenn rechts statt der $u$ die $x$ aus (1) gesetzt werden:

also:

$$
\sum v x=\sum a_{i r} x_{r} y_{i}
$$

and umgekehrt:

$$
v_{r}=\sum a_{i r} y_{i}
$$

$$
\Delta y_{i}=\sum \Delta_{1} v_{r}
$$

Soll demnach eiue Ebene $v$ den ihr entuprechenden Punkt enthalten, so muss:

$$
\Phi=\sum v_{r} v_{l} \Delta_{b r}=0
$$

sein. Die beiden Flächen $F^{2}$ und $\Phi$ stehen in der Beziduang zu cinander, dass $F$ darch die Substitution (1) in $\Phi$ übergeht und ungekeht $\Phi$ in $F$. In der That hat main:

$$
\Delta^{2} F=\sum a_{i r} u_{l} \Delta_{l i} u_{k} \Delta_{k r}=\Delta \sum u_{k} u_{t} \Delta_{i i}=\Delta \Phi,
$$

$F$ and $\Phi$ sind die bekannten beiden Ordnumysflächen*) aler Sulistitution (1).

Wir untersuchen nun, wie oft es geschieht, dass dem Punkte $x$ die Ebene $\mu$, dieser wieder der Punkt $x$ entspricht. Das heisst, os ist in den Gleichungen (1), (2), (4), (5) zu setzen:

$$
v_{i}=u_{i}, \quad q x_{i}=y_{i}
$$

So entstehen die Gleichungen:

d. h.:

$$
\left\{\begin{array}{l}
u_{i}=\sum a_{i} x_{r}, \\
u_{r}=e \sum a_{i r} x_{i},
\end{array}\right.
$$

$$
\vartheta \sum a_{i r} x_{i}-\sum a_{r i} x_{i}
$$

Die Gleichangen (7) führen auf eine Gleichung $q^{150}$ (irales in $\rho$, welche reciproke Wurceln besitct, deren Proluct gleich eins ist. Ist $n$ eine gerade Zabl, so sind in Algerneinen nur reciproke Wurkelu vor-

-) Vgl. Reye, Geometrio dur Latge II, 18. 
handen, während die Wurzel 1 nur als vielfache Wurzel anftreten kaw, für die mindestens noch alle ersten. Unterdeterminanten verschwinden. Ist dagegen $n$ ungerade, so ist eine Wurzel gleich 1 , die übrigen sind im Allgemeinen reciprok. ${ }^{*}$ )

Wir nehmen alle Wurzeln als reciprok $d$. h. $n$ als gerade an, sie sondern sich dann in zwei Systeme von je $s=\frac{n}{2}$ Wurzeln:

$$
\begin{aligned}
& r_{1}, r_{2}, \cdots r_{s}, \\
& \varrho_{1}, \varrho_{2}, \cdots \varrho_{s} ;
\end{aligned}
$$

und bezeichnen die Lösungen der Gleichung (7) durch

$$
\begin{aligned}
& x_{1}^{*}, x_{2}^{*} \cdots x_{n}^{*} \text {, gehörig zu } r_{s}, \\
& y_{1}^{*}, y_{2}^{*} \cdots y_{n}^{*}, \quad n \quad \text { zи } \varrho_{s} .
\end{aligned}
$$

Zwischen diesen Lösungen finden ganz ähnliche Bexiehungen statt, wie die in $\$ \mathrm{~J}$. behandelten. Zunächst ist aus (1):

also:

$$
r_{s} \sum a_{i k} x_{i}^{*}=\sum a_{k i} x_{i}^{s}
$$

$$
r_{s} \sum a_{i k} x_{i}^{s} x_{k}^{s}=\sum a_{k i} x_{i}^{s} x_{k}^{s} \text {. }
$$

Hieraus folgt, so lange nicht $r_{s}=1$ :

Ferner ist:

$$
\left\{\begin{array}{l}
\sum x_{i}^{s} x_{k}^{s} a_{i k}=0 \\
\sum y_{i}^{s} y_{k}^{s} a_{i k}=0
\end{array}\right.
$$

$$
\begin{aligned}
r_{s} \sum a_{i k} x_{i}^{*} x_{k}^{t} & =\sum a_{k i} x_{k}^{s} x_{k}^{t}, \\
r_{i} \sum x_{i}^{t} x_{k}^{*} a_{i k} & =\sum a_{k i} x_{i}^{t} x_{k}^{*},
\end{aligned}
$$

woraus, so lange $r_{3} r_{t}$ von Eins verschieden ist, folgt:

$$
\left\{\begin{array}{l}
\sum x_{i}^{s} x_{k}^{t} a_{i k}=0 \\
\sum x_{i}^{s} y_{k}^{t} a_{i k}=0 \\
\sum y_{i}^{s} y_{k}^{t} a_{i k}=0
\end{array}\right.
$$

Nur die Ausdrücke $\sum a_{i k} x_{i}^{*} y_{k}^{*}$ verschwinden nicht, sondern genügen den Gleichungen:

$$
\left\{\begin{array}{l}
r_{s} \sum x_{i}^{s} y_{k}^{*} a_{i 1}=\sum a_{k i} x_{i}^{s} y_{k}^{s}, \\
0_{s} \sum y_{i}^{s} y_{k}^{s} a_{i k}=\sum a_{k i} y_{i}^{s} x_{k}^{s} .
\end{array}\right.
$$

*) Die genauere Untersuchung aller möglichen Falle reciproker Substitutionen knüpft sich überhaupt damit an ganz ahnliche Betrachtungen an, wie sie in $\$$ II auseivander gesetat worden sind. 
Bezeichuet man den Ausdruck $\sum x_{i}^{*} y_{k}^{z} a_{i k}$ durch $\left[x_{x} y z\right]$, so bann mau die Gleichungen (8) (9) (10) in die folgenden zusammenfassen:

$$
\left\{\begin{aligned}
{\left[x_{s} x_{i}\right]=0, \quad\left[y_{s} x_{i}\right] } & =0, \quad\left[x_{s} y_{t}\right]=0, \\
r_{s}\left[x_{s} y_{s}\right] & =\left[y_{i} x_{i}\right]
\end{aligned}\right.
$$

Man hat ferner nach (2) und (5):

Die Gleichungen:

$$
\left\{\begin{array}{l}
\sum u_{i} \Delta_{i x}=\Delta x_{i}, \\
\sum u_{i} \Delta_{s i}=\Delta \rho x_{i} .
\end{array}\right.
$$

$$
\sum_{u_{i} \Delta_{i s}}=\sum u_{i} \Delta_{s}
$$

lieferu daher eine neue Determinante, welche dieselben Wurzelu besitat, wie die vorhin betrachtete. Dementsprechend finden awischen den Lösungen $u_{i}^{x}$ und den den entsprechenden reciproken Wurzeln zugehörigen $v_{i}^{x}$ diejenigen Gleichungen statt, welehe den (11) analog sind:

$$
\left\{\begin{array}{cl}
{\left[u_{s} u_{t}\right]=0,} & {\left[u_{s} v_{t}\right]=0, \quad\left[v_{*} u_{t}\right]=0} \\
{\left[u_{t} v_{s}\right]=\Delta\left[u_{3} y_{s}\right]}
\end{array}\right.
$$

Endlich hat man noch:

$$
\left\{\begin{array}{l}
\sum u_{i}^{*} x_{i}^{*}=\sum u_{i}^{s} y_{i}^{*}=\left[y_{s} x_{s}\right], \\
\sum v_{i}^{*} y_{z}^{\prime}=\sum x_{i}^{*} x_{i}^{*}=\left[x_{*} y_{s}\right],
\end{array}\right.
$$

Die Gleichungen (11)-(13) sagen aus:

Bei der reciproken Transformation simd (n geradej $n$ in ${ }_{2}^{\text {n }}$ pare

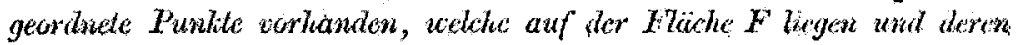

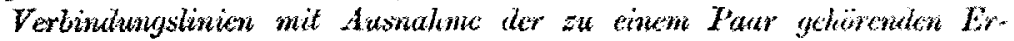

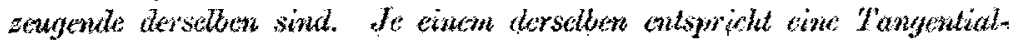

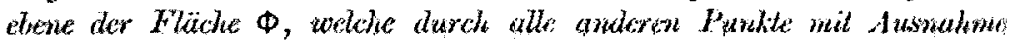

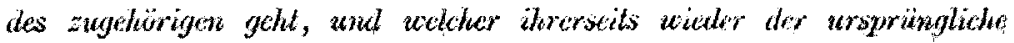
Pund als entsprechender vermäge der Transformation zugrorduat ist.

Bei ungeradem $n$ treten einige sehr leicht za erkennende Modificationen dieses Satzes auf.

Es wird angemessen sein, das aus jeuen punkten bestebende Gebilde zum Fundamentalgebilde einer Coordinatenbestimmung zu wählen. Wir führen daher die Transformation:

$$
x_{i}=\sum Y_{r} x_{i}^{r}+H_{r} y_{i}
$$

ein, welche an Stelle der Variabelen $x$ zwei Reihen neuer: 
A. Voss.

setzt. Dann ist:

$$
\begin{aligned}
& Y_{1}, Y_{2} \cdots Y_{s}, \\
& H_{1}, H_{2} \cdots H_{s},
\end{aligned}
$$

$$
\sum x_{i} u_{i}^{*}=\sum Y_{r} x_{i}^{*} u_{i}^{*}+H_{r} y_{i}^{\pi} u_{i}^{*}
$$

also nach (13)

$$
\left\{\begin{array}{l}
Y_{s}=\frac{\Sigma x_{i} v_{i}^{s}}{\left[x_{s} y_{s}\right]}, \\
H_{s}=\frac{\Sigma x_{i} u_{i}^{s}}{\left[y_{s} x_{s}\right]}
\end{array}\right.
$$

Führen wir ähnlich an Stelle der Ebenencoordinaten $v_{i}$ zwei Reihen $U_{i} V_{i}$ ein, so ist:

also:

$$
\sum(v x)=\sum Y U+H V=\sum \frac{\left(x_{i} v_{i}^{s}\right) U_{s}}{\left[x_{s} y_{s}\right]}+\sum \frac{\left(x_{i} u_{i}^{s}\right) V_{s}}{\left[y_{s} x_{s}\right]}
$$

oder:

$$
v_{i}^{\prime}=\sum \frac{V_{s} u_{i}^{s}}{\left[y_{s} x_{s}\right]}+\frac{v_{i}^{s} U_{s}}{\left[x_{s} y_{s}\right]}
$$

$$
\left\{\begin{array}{l}
U_{s}=\sum v_{i} x_{i}^{r} \\
V_{s}=\sum v_{i} y_{i}^{r}
\end{array}\right.
$$

Die Gleichungen (14)-(17) vermitteln die kanomische Gestalt der Reciprocität. Wir erhalten so zunächst die Formen $F$ und $\Phi$ in ihrer einfuchsten Gestalt. Und zwar ergiebt sich:

$$
F=\sum a_{i k} x_{i} x_{k}=\sum Y_{s} H_{s}\left(\left[x_{s} y_{s}\right]+\left[y_{s} x_{s}\right]\right)
$$

sowie:

$$
\Phi=\sum \Delta_{i k} u_{i} v_{k}=\Delta \sum V_{s} U_{s} \frac{\left(\left[x_{s} y_{s}\right]+\left[y_{s} x_{s}\right]\right)}{\left[x_{s} y_{s}\right]\left[y_{s} x_{s}\right]}
$$

Aus den Gleichungen (18) (19) geht unmittelbar hervor, dass die Gleichung $\Phi$ in Punktcoordinaten geschrieben wieder von der Form (18) ist, d. h. dass die Fläche $F$ von $\Phi$ in dem aus Erzeugenden gebildeten Fundamentalgebilde geschnitten wirl*).

Wir gehen nun dazu über, die kanowische Form der recimolen Substitution (1) selbst herzuleiten. Da:

$$
\begin{aligned}
u_{i} & =\sum a_{i r} x_{r}=\sum a_{i r} Y_{s} x_{r}^{s}+a_{i r} H y_{r}^{*} \text { wach }(1),(14), \\
& \left.=\sum v_{i}^{*} v_{s} x_{s}\right]+\frac{v_{i}^{*} v_{s}}{\left[x_{s} y_{s}\right]} \text { nach }(16),
\end{aligned}
$$

J Vgl, Sehroter, Crelle 77, pag, 140 ft. 
so ergiebt sich, wenn man mit $x_{i}^{*}$ moltiplicirt:

$$
H_{t}\left[x_{t} y_{t}\right]=U_{t},
$$

und ebenso durch Multiplication mit $y_{i}^{\prime}$ :

$$
Y_{t}\left[y_{t} x_{i}\right]=V_{t} \text {. }
$$

Darnach sind:

$$
\left\{\begin{array}{l}
{\left[x_{t} y_{t}\right] H_{t}=U_{t}} \\
{\left[y_{t} x_{t}\right] Y_{t}=V_{t}}
\end{array}\right.
$$

die kanonischen Formeln für die allgemeine reciproke Substitution.

Man kann hiernach formen zweiter Ordnung angeben, welche durch die reciproke Substitution in sich ibergehen.

Setzt man zur Abkürung $\left[x_{i} y_{i}\right]\left[y_{i} x_{i}\right]$ gleich $a_{l}$, so wird die Punktfläche:

$$
f=\sum Y_{t} \mathrm{H}_{i} \lambda_{t}=0
$$

nach $(20)$ übergehen in die Tangentenebenentläcbe

$$
\varphi=\sum U_{t} V_{i}^{a_{i}}=0,
$$

welche selbst wieder mit der Punktfäche (forma adjuncta)

$$
f^{\prime}=\sum Y_{t} H_{t} \frac{a_{t}}{2_{t}}=0
$$

gleichbedeutend ist. Daher shod die Bedingungen, unter welchen $f$ in sich selbst äbergeht:

$$
\lambda_{t}^{2}=a_{t}
$$

und der allgemeine Ausilruck des Systems, dessen sämmtliche Individuen in sich ubergehen, ist:

$$
\Omega=\sum Y_{i} H_{t} / a_{t}
$$

wo die Quadratwurzelu mit beliebigen Zeichen genommen werdeu können. $\Omega$ ist eine Gruppe in der Mannigfaltigheit der Formek, dio gegenseitig in sich übergehen; in der That müsson je znci Formen, die überhaupt in dem System

$$
\sum Y_{t} H_{i} \lambda_{i}
$$

enthalten sind, durch die reciprohe Substitution in dieser Weise cinander zugeordnet erscheinen. Für $n=4$ erhalten wir also aus (22) zwei Flächen, die in sich übergehen und zu dem Büschel gehören, von dem die beiden Ordnungstlüchen $F, \Phi$ zwei Individuen sind. Je zuei Flëcken dieses Büscluels gehen in Allgmeinon gegenseitig in anawder über, vählrend die beiden: 


$$
\begin{aligned}
& Y_{1} H_{1} \sqrt{a_{1}}+Y_{2} H_{2} \sqrt{a_{2}}, \\
& Y_{1} H_{1} \sqrt{a_{1}}-Y_{2} H_{2} \sqrt{a_{2}},
\end{aligned}
$$

in sich selbst transformirt werden.

Für ungerade Variabelnzahl würde man dagegen dem der Wurzel 1 zugehörigen Punkte $x_{i}^{\prime}$ entsprechend den Gleichungen (15) (17) (20) noch die folgenden respective:

$$
\begin{aligned}
& Y_{1}=\sum \frac{x_{i} u_{i}^{\prime}}{\left[x_{i} x_{1}\right]}(15), \\
& U_{1}=\sum u_{i} x_{i}^{\prime}(17), \\
& Y_{1}=\frac{U_{1}}{\left[r_{1} x_{1}\right]}(20)
\end{aligned}
$$

hinzuzufügen haben. Die Form:

wïrde darnach in

$$
Y_{1} \lambda_{1}+\sum Y_{i} H_{i} \lambda_{1}
$$

$$
U_{1}^{2} \frac{\lambda_{t}}{a_{1}^{2}}+\sum U_{t} V_{t} \frac{\lambda_{t}}{a_{t}}=Y_{1}^{2} \frac{a_{1}^{2}}{\lambda_{1}}+\sum Y_{t} H_{t} \frac{a_{t}}{\lambda_{t}}
$$

übergetührt werden. Fïr $n=3$ gehen also je zwei Curven des Büschels sich doppelt berïhrender Kegelschnitte

$$
Y_{1}^{2} \lambda_{1}+\lambda_{2} H_{2} Y_{2}=0
$$

gegenseitig in einander ïber, während die beiden besonderen Kegelschnitte in sich selbst transformirt werden.*)

$$
Y_{1}^{2} a_{1} \pm Y_{2} H_{2} \sqrt{a_{2}}
$$

\section{\$ VIII.}

Die Transformation einer Fläche zweiten Grades in sich selbst.

Wir wollen hier die Transformation einer gegebenen Fläche zweiten Grades in sich noch kurz besprechen. Dabei ist die Gleichung derselben in Limiencoordinaten vorausgesetzt worden, eine Auffassung, die im Ganzen vio Classen zon Transformationen als gleichwerthig hervortreten lässt.

Die beiden Systeme der Erzeugenden einer Fü̈che zweiten Grades lassen sich in der Form: ${ }^{* *}$ )

$$
\left\{\begin{array}{l}
x_{i}=\mu^{2} a_{i}+b_{i} \lambda \mu+c_{i} \lambda^{2} \\
y_{i}=M^{2} \alpha_{i}+\beta_{i} \wedge M+\gamma_{i} \Lambda^{2}
\end{array}\right.
$$

darstellen, wo $x, y$, die Liniencoordinaten derselben, Functionen zwei-

-) Rosanes, Crelle 80, pag. 67, Aumerkung.

*) Voss, Math Anualen VII, 118. 
ten Grades der homogenen Paraneter $\lambda, \mu ; \Lambda, M$ sind. Diluei müssen die Gleichungen:

$$
\begin{gathered}
\left(a^{3}\right)=0, \quad(b a)=0, \quad(b c)=0, \quad\left(c^{3}\right)=0, \\
2(a c)+(b)=0,
\end{gathered}
$$

und die analogen fur die $\alpha_{i}, \beta_{i}, \gamma_{i}$ vorausgesetat werden, während:

$$
(\alpha a)=0,(a b)=0, \quad(\alpha c)=0, \quad(\beta a)=0, \cdots \text { u. s. w. }
$$

ist. Die Gleichung dex Fläche selbst erscheint in der queifachen Gestalt:

$$
\left\{\begin{array}{l}
F=4 a_{x} c_{x}-b_{x}^{2}=0, \\
F^{\prime}=4 \alpha_{x} \gamma_{x}-\beta_{x}^{2}=0,
\end{array}\right.
$$

wobei $F, F^{\prime}$ durch die Identitit:

$$
\sum x_{i}^{2}+\frac{k^{\prime}}{\left.b^{*}\right)}+\frac{F^{\prime}}{\left(h^{2}\right)}=0
$$

verbunden sind. Das Verbältniss:

$$
\frac{\left(u^{2}\right)}{\left(A^{*}\right)}=\frac{(a c)}{(a y)}
$$

mag mit $E$ bezeichuet werden.

Setzt man an Stelle der $\lambda_{r}, \mu$ oder $\Lambda, M$ die Ausdrücke $a \mu+b \lambda$, $e \mu+d \lambda$ oder $\alpha M+\beta \Lambda, \gamma M+\delta \Lambda$, so werden die Erzengendep projectivisch in $x^{\prime}, y^{\prime}$ transformirt, wo dann:

$$
\left\{\begin{array}{l}
x_{i}^{\prime}=(c \mu+d \lambda)^{2} a_{i}+b_{i}(\alpha \mu+b \lambda)(c \mu+\alpha \lambda)+c_{i}(\alpha \mu+b \lambda)^{2} \\
y_{i}^{\prime}=(\gamma M+\delta \Lambda)^{2} a_{i}+\beta_{i}(\alpha M+\beta \Lambda)(\gamma M+\alpha \Lambda)+\gamma_{0}(\alpha M+\beta \Lambda)^{2}
\end{array}\right.
$$

Da ferner jede Tangente der Flïche in der Form $x_{i}+y y_{i}$ erscheint, so kann die Transformation des Tangentensystenes nur dariq bestehen, dass an Stelle der Tangente $x_{i}+\nu y_{i}=z_{\text {d }}$ die neue $z_{i}^{\prime}=l x_{i}^{\prime}+m y_{i}^{\circ}$ tritt. Da nun aus (1):

$$
\begin{array}{ll}
a_{i}=\lambda^{2}(a), & \alpha_{i}=\Lambda^{2}(\alpha \gamma), \\
b_{z}=\lambda \mu\left(b^{2}\right), & \beta_{i}=\Lambda \mathrm{M}\left(\beta^{2}\right), \\
\left.c_{z}=\mu^{2}(\alpha)\right), & \gamma^{2}=\mathrm{M}^{2}(\alpha \gamma),
\end{array}
$$

so kann man vermöge $z_{i}^{\prime}=l x_{i}^{\prime}+m y_{i}^{\prime}$ die formen $a_{z}^{\prime}, b_{s}^{\prime} \cdots$ leicht durch die $z$ ausdrücken, und erbült sis die Trasusormationsformoln:

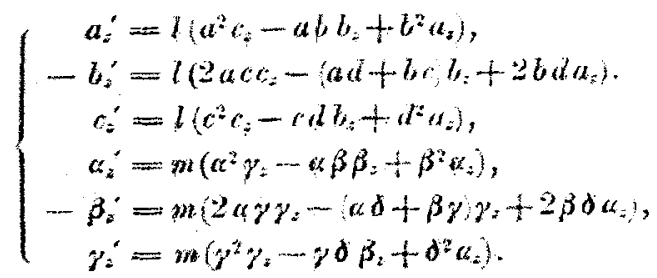

Man kann in derselben $l=m=1$ voraussetzen. Da feruks: 


$$
\begin{aligned}
& 4 a_{z}^{\prime} c_{z}^{\prime}-b_{z}^{2}=(a d-b c)^{2} F, \\
& 4 \alpha_{z}^{\prime} \gamma_{z}^{\prime}-\beta_{z}^{\prime 2}=(\alpha \delta-\beta \gamma) F^{\prime}
\end{aligned}
$$

so ist nach (3) erforderlich, wenn die Substitution (4) überhaupt eine orthogonale sein soll, dass:

$$
(a d-b c)^{2}=(\alpha \delta-\beta \gamma)^{2}
$$

und also die Werthe der beiden Determinanten $(a d-b c),(a \delta-\beta \gamma)$, 7 and $x$ so ist:

and zugleich:

$$
k^{2}=x^{2}
$$

$$
\left\{\begin{array}{l}
\sum_{z_{1}^{2}}=k^{2} \sum_{z^{2}}, \\
\sum_{z_{1} y_{1}}=k^{2} \sum z y .
\end{array}\right.
$$

Die Determinante der Substitution (4) ist gleich $k^{3} x^{2}$, und die Transformation ist demnach eigentlich oder uneigentlich, je nachdem $k=x$ oder $k=-x$ ist.

Es giebt noch eine zweite Transformation der Fläche; sie entsteht, wenn man zugleich eine Vertauschung der beiden Systeme von Erzeagenden ausführt, d. h. wenu man in (4) rechter Hand die lateinischen Buchstaben mit den entsprechenden griechischen vertauscht. Für sie gelten daher die Formeln:

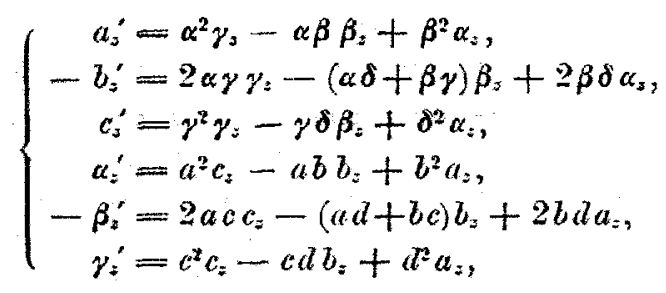

wobei wieder:

also

$$
\begin{aligned}
& 4 a_{z}^{\prime} c_{z}^{\prime}-b_{z}^{\prime 2}=(\alpha \delta-\gamma \beta)^{2} F^{\prime}, \\
& 4 \alpha_{z}^{\prime} \gamma_{s}^{\prime}-\hat{p}_{z}^{\prime 2}=(a d-b c)^{2} F,
\end{aligned}
$$

$$
(\alpha \gamma-\gamma \beta)^{2} \varepsilon^{2}=(a d-b c)^{2}
$$

vorausgesetzt werden muss. Die Determinante der Substitution (6) ist - $k^{3} x^{3}$ und die letztere daher eigentlich oder uneigentlich, je nachdem $k+\varepsilon x=0$ oder $k-\varepsilon x=0$. Die formeln (4), (6) stellen zugleich die allgemeinsten Transformationen des Linienraumes vor, bei denen eine Fläche zweiten Grades in sich übergeht.

Wir betrachten zanächst die Gleichungen (4).

Zur Bestimmung der linearen Complexe, welche durch die Transformation in sich abergefuhrt werden, hat man zu setzen: 


$$
\begin{aligned}
& k_{1} a_{2}^{\prime}+k_{2} b_{2}^{\prime}+k_{3} c_{2}^{\prime}=\sigma\left(k_{1} \alpha_{2}+k_{3} b_{2}+k_{3} c_{2}\right), \\
& x_{1} \alpha_{2}^{\prime}+x_{2} \beta_{3}^{\prime}+x_{3} \gamma_{2}^{\prime}=\sigma\left(x_{1} \alpha_{3}+x_{3} \beta_{3}+x_{3} \gamma_{2}\right) .
\end{aligned}
$$

Für die $k_{1}, k_{2}, k_{3}$ entstehen so die Gleichungen:

$$
\left\{\begin{array}{l}
e k_{1}=k_{1} b^{2}-2 b a k_{2}+k_{3} k_{2}^{2} \\
\rho k_{2}=-k_{1} a b+(a b+b c) k_{3}-k_{3} e d, \\
\rho k_{3}=k_{1} a^{2}-2 a c k_{2}+k_{3} a^{2}
\end{array}\right.
$$

also zur Bestimmung von $\varrho$ die cubische Gleichung:

oder:

$$
\left|\begin{array}{ccc}
b^{2}-e & -2 b a & d^{2} \\
-a b & a d+b c-e & -c d \\
a^{2} & -2 a c & c^{2}-e
\end{array}\right|=0
$$

$$
(\varphi+k)\left[(\varphi-k)^{2}-n^{2} \varphi\right]=0
$$

wo:

$$
n=b+c \text {. }
$$

Ganz analog hat man zur Bestimmung von $x_{1}, x_{2}, x_{3}, \sigma$ die Gleichung:

oder :

$$
\begin{array}{ccc}
\beta^{2}-\sigma & -2 \beta \delta & \delta^{2} \\
-\alpha \beta & \alpha \delta+\beta \gamma-\sigma & -\gamma^{\delta} \\
a^{2} & -2 \alpha \gamma \quad y^{2}-\sigma
\end{array}=0,
$$

$$
\begin{gathered}
(\sigma+x)\left[\left(\sigma-x^{2}-\nu^{2} \sigma\right]\right]=0 \\
\nu=\beta+\gamma
\end{gathered}
$$

Bezeichnet man die den drei Wurzeln $\theta_{1}=-7, \varphi_{1}, \varrho_{2}$ entspreatenden linearen Complexe durch $A_{z}, b_{z}, C_{z}$, dio den Wurzeln $\sigma=-\infty$, $\sigma_{1}, \sigma_{2}$, zuggehörigen durch $\mathrm{A}_{*}, \mathrm{~B}_{i}, \boldsymbol{\Gamma}_{*}$, so hat man die Identitäten:

$$
\left\{\begin{array}{l}
A_{i}^{\prime}=-B_{i} A_{i}, \\
B_{z}^{\prime}=Q_{1} B_{i}, \\
C_{z}^{\prime}=Q_{2} C_{i}, \\
A_{z}^{\prime}=\cdots A_{i}, \\
B_{z}^{\prime}=\sigma_{1} B_{i}, \\
\Gamma_{i}^{\prime}=\sigma_{z} \Gamma_{2},
\end{array}\right.
$$

Die Complexe $B_{i}, C_{*}, \mathrm{~B}_{i}, \Gamma_{*}$ sind specille, sie stellen zwei Pare von Erzeugrenden vor, welche bei der Transformation fest bleiben. Dagegen hat man für $A_{z}, \mathrm{~A}_{z}$ die Gleichungen:

$$
\left\{\begin{array}{l}
A_{i}=a_{i} d+\frac{b-c}{2} b_{i}-a c_{i j} \\
\mathrm{~A}_{i}=\alpha_{i} d+\frac{\beta-\gamma}{2} \beta_{z}-a \gamma_{z}
\end{array}\right.
$$

und diese Complexe sind yur dann speciell, wenn $n^{2}-4 k=0$ oder 
$\nu^{2}-4 x=0, \mathrm{~d} . \mathrm{h}$. wern die Discriminante der quadratischen Factoren von (9) and (i1) verschwindet.

Ist nun $k=x$, so geht gleichzeitig jeder Complex des Büschels:

$$
A_{z}+\rho A_{z}=0
$$

in sich über, und die beiden in letzterem befindlichen speciellen Complexe sind die beiden Diagonalen des festen Erzeugenden-Vierseites: die Transformation ist (im Sinne der Punktgeometrie) eine eigentliche collineare. $([11,1,1,1,1])$

Wenn dagegen $z=-x$, so geht $A_{z}+\varrho \mathrm{A}_{z}$ über in $A_{z}^{\prime}-\varrho \mathrm{A}_{z}^{\prime}$, d. k. je zwei Complese jenes Büschels, also auch die beiden Diagonalen vertauschen sich gegenseitig, und die Transformation ist eine reciprolit $([1,1,1,1,1,1])$.

Wird ferner die Transformation (6) zu Grunde gelegt, so kann man verlangen, dass die lineare Form:

$$
\begin{array}{r}
k_{1} a_{z}^{\prime}+k_{2} b_{z}+k_{3} c_{3}{ }^{\prime}+k_{4} \alpha_{z}^{\prime}+k_{5} \beta_{z}^{\prime}+k_{6} \gamma_{z}^{\prime} \\
=\rho\left(k_{1} a_{z}+k_{2} b_{z}+k_{3} c_{z}+k_{3} \alpha_{z}+k_{5} \beta_{z}+k_{6} \gamma_{z}\right)
\end{array}
$$

werde. Zur Bestimmung dex $k$ hat man die 6 Gleichungen:

$$
\left\{\begin{array}{c}
\rho k_{1}=b^{2} k_{4}-2 b d k_{5}+d^{2} k_{6}, \\
-\varrho k_{2}=a b k_{4}-(a d+b c) k_{5}+a c k_{4}, \\
\varrho k_{3}=a^{2} k_{4}-2 a c k_{5}+c^{2} k_{6}, \\
\varrho k_{4}=\beta^{2} k_{1}-2 \beta \delta k_{2}+\delta^{2} k_{3}, \\
-\varrho k_{5}=\alpha \beta k_{1}-(\alpha \delta+\beta \gamma) k_{2}+\delta \gamma k_{3}, \\
\rho k_{6}=\alpha^{2} k_{1}-2 \alpha \gamma k_{2}+\gamma^{2} k_{3},
\end{array}\right.
$$

welche für $\rho^{2}$ die cubische Gleichung:

$$
\left|\begin{array}{ccc}
A^{2}-\rho^{2} & -2 A B & B^{2} \\
-A C & (A D+B C)-\rho^{2} & -B \\
C^{2} & -2 C D & D^{2}-\rho^{2}
\end{array}\right|=0
$$

liefern, in welcher:

Setzt man:

$$
\begin{array}{ll}
A=b \beta+\alpha d, & C=a \beta+\alpha c \\
B=b \delta+\gamma d, & D=c \gamma+a \delta .
\end{array}
$$

$$
\begin{aligned}
& K=A D-B C=(a d-b c)(\alpha \delta-\beta \gamma), \\
& \mathrm{N}=A+D=\beta b+a d+a \delta+c \gamma,
\end{aligned}
$$

so hat man:

$$
\left(\rho^{2}-K\right)\left[\left(\rho^{2}+K\right)^{2}-N^{2} \rho^{2}\right]=0 \text {. }
$$

In diesem Falle hat also die Determinante $\Omega(\varrho)$ nur Potenzen von $\varrho$ mit geralem Exponenten (vgl. \$ III, S. 335).

Eine vollständige Untersuchung der Elementartheiler von $\Omega(\varphi)$ 
für alle speciellen Falle, welche die Transformationen (4) (6) darbieten können, wärde hier zu weit führen. Um aber wenigstens den allgemeinen Fall, in welchem die Wurzeln der bezüglichen Gleichungen (9), (11), (16) von einander verschieden sind, genauer zu übersehen, wollen wir zunächst bei eigentlicher Transformation in (4):

$$
a=0, \quad d=0, \quad \alpha=0, \quad \delta=0, \quad b c=\beta \gamma=k
$$

setzen. Darnach bleiben die Erzeugenden:

$$
a, \gamma, a, c
$$

und das Būschel $b_{3}+\rho \beta_{z}$ fest. Es müssen daher alle Flächen zweiten Grades mit dem Parameter $\rho$ :

$$
\Psi=F\left(\rho^{2}-\varepsilon\right)+2 \rho \varepsilon\left[2 \rho \alpha_{*} \gamma_{s}+b_{*} \beta_{*}\right]=0
$$

in sich übergehen. Jede Gerade $z$ des Raumes transformirt sich hiernach als Tangente an $2 w e i$ der Flächen des Büschels $\Psi$, deren Parameter bei gegebenem $z$ durch die Gleichnng (17) bestimmt werden.*)

Da die Fläche $F=0$ keine besondere Stelle in jenem Bilsehel einninmt, so genügt es $z$ als Tangente vorauszusetzen, d. h.:

$$
\begin{array}{ll}
a_{1}=l, & a_{s}=m, \\
b_{1}=2 l \lambda, & \beta_{s}=2 m \mu, \\
c_{s}=\lambda^{2} l, & \gamma_{2}=m \mu^{2},
\end{array}
$$

anzunebmen. Jede Tangente von $F$ ist daher gleichzeitig Tangente an diejenige Fläche $\psi=0$, für welche:

$$
\varphi=-\frac{2 l 2}{m \mu} \text {. }
$$

*) In Verbindang bierwit steht der Satz, den Herr Cayley (Pbil. Magazine 1953, VI, 326) bemerkt bat: Die Verbindungsinien correspovidirender Punkte der Fläche siud Tangenten an zwei feste Flächen zateiten Grades. Will man diesep Satz direct beweisen, so transformire man die Fläche:

$$
x_{1} x_{2}=\omega=-x_{2} x_{4}
$$

durch die Gleichungen $y_{i}=\lambda_{i} r_{i}$ in sich, wo $\lambda_{1} \lambda_{3}=\lambda_{2} \lambda_{4}$. Die Liniencoordinatep einer Verbindungsgeraden $\{x y\}$,

stehen in der Beziehung, dass:

$$
p_{1}, p_{3,}, p_{3}, p_{4}, p_{3}, p_{6}
$$

$$
\frac{p_{2}^{2}}{\left(\lambda_{2}-\lambda_{2}\right)^{2}}=\frac{p_{1}^{2}}{\left(\lambda_{4}-\lambda_{2}\right)^{2}}=\frac{p_{3} p_{1}}{\left(\lambda_{1}-\lambda_{4}\right)\left(\lambda_{2}-\lambda_{2}\right)}=\frac{p_{1} p_{4}}{\left(\lambda_{1}-\lambda_{2}\left(\lambda_{4}-\lambda_{2}\right)\right.}
$$

Da aun die Gleichang des Fuchentiachels $x_{1} x_{2}+k x_{2} x_{1}=0$ wit den namblichen vier featen Erzeagenden in Liritencoordinaten ist:

$$
p_{a}^{2}+k^{2} p_{2}{ }^{2}+2 k\left(p_{3} p_{6}-p_{1} p_{0}\right)=0
$$

so erkennt man obne Weiteres, dass die Gleichung:

$$
k^{2}\left(\lambda_{2}-\lambda_{2}\right)^{2}+z k\left[\left(\lambda_{1}-\lambda_{4}\right)\left(\lambda_{3}-\lambda_{2}\right)-\left(\lambda_{1}-\lambda_{3}\right)\left(\lambda_{4}-\lambda_{3}\right)\right]+\left(\lambda_{4}-\lambda_{2}\right)^{2}=0
$$

die Parameter $k_{i}, k_{2}$ der genannten beiden Flacheu de Büschel bentimmt. 
Andererseits sind uberhaupt die Geraden $z$, welche von ihren transformirten $z$ geschnitten werden, durch die Gleichung:

$$
\left(4 A_{z}{ }^{2}-\left(n^{2}+2 k\right) F\right)+\varepsilon\left(4 \Delta_{z}{ }^{2}-\left(\nu^{2}+2 K\right) F^{\prime}\right)=0
$$

bestimnat, worans in unserem speciellen Falle und für $F=F^{\prime}=0$ wird:

$$
l^{2} \lambda^{2}(b-c)^{2} \pm \varepsilon m^{2} \mu^{2}(\beta-\gamma)^{2}=0
$$

d. 7.: die Tangenten von $F$, welche von ihren Transformirten geschnitten werden, sind durch zwei verschiedene lineare Complexe ausgeschieden und zerfallen darnach in zwei getrennte Systeme: die sämmtlichen Geraden eines jeden beriïhren nach (18), (19) noch je eine feste Fläche des Büschels $\psi$.

Ist dagegen $\beta_{\gamma}=-b c$, so ist die Transformation eine reciproke, und das Busschel $\psi$ hat die Eigenschaft, dass je zwei Flächen dessellen gegenseitig in einander übergehen, wühtrend zwei Fläckien dessellen, nämlich die gegebene (für $\varrho=0$ ) und die Flüche $\varepsilon \beta_{x}^{2}-4 a_{x} c_{x}=0$ (fïr $\rho=\infty$ ) fest bleiben.

Betrachten wir zweitens die aus (6) sich ergebenden formeln:

$$
\begin{array}{ll}
a_{z^{\prime}}=\beta^{2} \alpha_{z}, & \alpha_{z^{\prime}}=b^{2} a_{z_{2}} \\
b_{z^{\prime}}=\beta \gamma \beta_{z}, & \beta_{z^{\prime}}=b c b_{z}, \\
c_{z^{\prime}}=\gamma^{2} \gamma_{z}, & \gamma_{z^{\prime}}=c^{2} c_{z},
\end{array}
$$

wenn wieder $a=d=\alpha=\beta=0$ gesetzt wird. Ist nun:

$$
\beta \gamma+\varepsilon b c=0 \text {, }
$$

so sind sämmtliche in sich ïbergehende lineare Complexe:

$$
\left\{\begin{aligned}
b a_{z^{\prime}} \pm \beta \alpha_{i} & = \pm b \beta\left[b a_{z} \pm \beta \alpha_{z}\right] \\
c c_{z} \pm \gamma \gamma_{i} & = \pm c \gamma\left[c c_{i} \pm \gamma \gamma_{s}\right] \\
\sqrt{b c} b_{z^{\prime}} \pm \sqrt{\beta \gamma} \beta_{z^{\prime}} & = \pm \sqrt{\beta \gamma b c}\left[\gamma / \bar{b} c b_{z} \pm \gamma \overline{\beta \gamma} \beta_{s}\right]
\end{aligned}\right.
$$

specielle und bestimmen die sechs Kanten eines Tetraeders, von welchen vier Tangenten der Fläche sind: die Transformation ist eine wneigentliche collineare. Bezeichnet man jene sechs Complexe (20) durch

$$
A_{*}, B_{s}, C_{z}, \mathrm{~A}_{z}, \mathrm{~B}_{z}, \Gamma_{i} \text {, }
$$

so gehen in Folge der Gleichungen (20) die linearen Formen der Gruppen:

$$
\left\{\begin{array}{rr}
A_{z}+\rho \mathrm{A}_{z}, & A_{z}-\rho \mathrm{A}_{s}, \\
\text { a) } B_{s}+\sigma \mathrm{B}_{z}, & \text { b) } B_{z}-\sigma \mathrm{B}_{z}, \\
C_{z}+\tau \Gamma_{z}, & C_{z}-\tau \Gamma_{s},
\end{array}\right.
$$

gegenseitig in einander äber. Gleiches wird daher von den durch sie erzeugten $\infty^{3}$ Flächen zweiten Grades gelten. Im Allgemeinen ist die durch die Grappe a) erzengte Flāche eine andere, wie die ans b) ent- 
stehende. Aber es entsteht die nämliche flüche, wenn simmiliche Complexe a) wit deuen von b) in Inrolution liegen.

Dies fuhrt, wegen $\beta \gamma+\varepsilon b c=0$, und $\mathrm{da}$ :

$$
\begin{aligned}
& A_{1}+\rho A_{z}=\beta \alpha_{2}(1+\rho)+b \alpha_{i}(1-\varphi), \\
& B_{z}+\sigma B_{s}=\gamma \gamma_{3}(1+\sigma)+c c_{2}(1-\sigma), \\
& c_{2}+\tau \Gamma_{s}=\sqrt{\beta \gamma} \beta_{s}(1+\tau)+\sqrt{\phi c} b_{s}(1-\tau) \text {, }
\end{aligned}
$$

auf die einzige Bedingung:

$$
\sigma=\rho
$$

wonach also ein ganses Netz von Flächen zaeiten Crrales fos bleilt.

Hat man dagegen $\beta \gamma-\varepsilon b c=0$, so befinden sich unter den Complexen (20) zwei nicht specielle, die Transformation ist eine reciproke. Auch hier gehen $\infty^{*}$ Flächen gegenseitig in sich uber, aber es existirt nur eine einfach unendliche schaar in sich fester Flachon, weil die Bedingungen:

$$
\rho x=1, \quad \sigma=1,
$$

erforderlich sind, wenn sämmtliche Complexe der Gruppe a) mit denen von Gruppe b) in Involution liegen sollen.

\section{$\$ x$.}

Orthogonale Transformation linearer Formen in sich selbst,

Eine eigentliche Substitution transformirt im Allgemeinen nur specielle lineare Formen $a_{x}$ in sich, $d . h$. solche, deren Coefficiention der Bedingung $\sum a_{i}{ }^{2}=0$ genügen. Wird aberhaupt eine allgemeine lineare Form in sich transformirt, so ist dies gleich mit einem Bischel solcher Formea der Fall. Anders ist es mit der uneigentlichen Sub. stitution: sie transformirt schon im Allgemeinen zwei, aber auch nur zwei lineare Formen allgemeinen Charakters in sich, während die Individueu des ans ihnea gebildeten Bijschels gegenseitig vertauscht werden.

Um tormeln anzugeben, welche eine gegebene Form $a_{x}=0$ orthogonal in sich transformiren, construire man eine Gruppe von ${ }_{z}^{n}$ Funktpaaren $x_{i}^{*}, y_{i}^{*}\left(s=1,2 \cdots \frac{n}{2}\right)$, welche auf der Fläche $r=\sum x_{i}^{2}=0$ liegen, und deren Verbindungslinien ad je zweien Erzeugende der Fläche sind, mit Ausnahme derer, welche zu einem Pare gehören. Die Punkte $x_{i}^{\prime}, y_{i}^{\prime}$ wähle man so, dass:*)

* In ganz anlicher Weise kann man sutstitutionen bilden, felche 2 wei and mebr lineare Formen in sich transformiren. Inbesoudere wei her woch dis (speciclie) Subatitution

$$
y_{i}=F_{i}
$$




$$
y_{i}^{\prime}=x_{i}^{\prime}-2 a_{x}^{\prime} \frac{a_{i}}{\Sigma a_{i}^{2}}
$$

die übrigen unterwerfe man ausserdem den Bedingungen:

$$
a_{x}^{*}=0, \quad a_{y}^{*}=0,
$$

und ordue den Punkten $x^{\prime}, y^{\prime}$ die Zahl 1 , den übrigen $x_{i}^{*}, y_{i}^{s}$ Zahlen $r_{a}, Q_{a} z \mathfrak{a}$, deren Product gleich 1 ist. Dann wird die Transformation mit den Coefficienten

$$
b_{i k}=x_{i}^{\prime} y_{k}^{\prime}+x_{k}^{\prime} y_{i}^{\prime}+\sum r_{s} x_{i}^{s} y_{k}^{s}+e_{s} x_{k}^{s} y_{i}^{s}, \quad s=1 \ldots \frac{n-2}{2},
$$

nack \& IOI. die verlangte Eigenschaft besitzen. Und sie ist zugleich die allgemeinste Transformation dieser Art, da sie $\frac{1}{2}(n-1)(n-2)$ willkürliche Parameter enthäl.

Will man dagegen Formeln haben, welche die erforderliche Zahl von expliciten Parametern enthalten, so scheint es am einfachsten, die gegebene Form zuerst orthogonal in $y_{n}=0$ (etwa durch eine specielle uneigentliche Transformation, wie im Folgenden gezeigt wird) und dann $\sum y^{2}-y_{n}{ }^{2}$ durch die Cayley'schen Formeln in sich zu transformiren.

Man kann aber auf folgendem Wege einfachere Formeln erbalten, die zwar nicht allgemein sind, aber bei manchen Gelegenheiten eine vortheilhaftere Anwendung finden.

Die specielle uneigentliche Substitution:

$$
x_{i}=y_{i}-2 \alpha_{i} \frac{\alpha_{y}}{\sum \alpha_{i}^{2}}
$$

transformirt den Ausdruck $a_{x}$ in sich selbst, wenn $(a \alpha)=0$. Sie stellt deminach $0^{-1-z}$ uneigentliche specielle Substitutionen der verlangten Eigenschaft dar.

Andererseits bedingen die beiden speciellen uneigentlichen Substitutionen:

die Identitäten:

$$
\begin{gathered}
x_{i}=y_{i}-\frac{\left(\alpha_{y} \pm m \beta_{y}\right)}{(\alpha \alpha) \pm m(\alpha \beta)}\left(\alpha_{i} \pm m \beta_{i}\right), \\
m=\sqrt{\frac{(\alpha \alpha)}{(\beta \hat{\beta})}}
\end{gathered}
$$

$$
\left\{\begin{array}{l}
\alpha_{x}=\mp m \beta_{y}, \\
\beta_{x}=\mp \frac{1}{m} \alpha_{y} .
\end{array}\right.
$$
(Seite 29 ) erwahnt, welche enichtlich $\frac{n}{2}$ willkürliche lineare Formen $a_{x}, b_{x}, \ldots$
in sinh nherfinhr. 


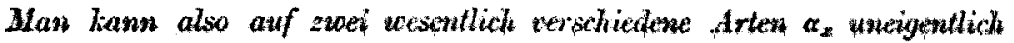
in die willüurliche Form $\beta_{s}$ transformiren. Wendet man die beiden (vertauschbaren) Substitutionen (3) nach einunder an, so entsteht eine eigentliche Substitution, welche $a_{x}$ in sich transformirt:

(5) $x_{i}=y_{i}-\frac{\left\langle\alpha_{y}+m \hat{\beta}_{y}\right)}{(\alpha \alpha)+m(\alpha \beta)}\left(\alpha_{i}+m \beta_{i}\right)-\frac{\left(\alpha_{y}-m \hat{\beta}_{y}\right)}{(\alpha \alpha)-m(\alpha \beta)}\left(\alpha_{i}-m \beta_{i}\right)$.

Die Formel (5) stellt mithin $\infty^{n-1}$ eigowliche Substitutionen vor, wcelch" $a_{x}$ in sich transformiren. Wendet man die Transformation (5) zweimal hintereinander an, so entsteht wieder eine identische Transformation.

Allgemeinere Formeln entstehen, wenn man verschiedene Substitutionen der Art (1) ader (5) oder beide unter einander in irgend einer Weise combinirt. Insbesondere aler erschäpt shon aie Formal (5) alle Transformationen eines auf der Flüde $f=x_{1}{ }^{2}+x_{2}{ }^{2}+x_{3}{ }^{2}+x_{4}{ }^{2}=0$ gelegenon Kegelschnittes in gevöhnlichen fiaume in sich sellst. Der greometrische Inhalt derselben ist folgender. Wurch die frorm $\beta_{x}=0$ wird dem hesgelschnitte $K$, in welehem $f$ von $\varkappa_{x}=0$ gesehnitten wird, ein Kegrelsctnitt $K^{\prime}$ adjungirt. Die I'ranstormation (5) geht nun aus awei Centralprojectionen hervor, deren Centra die beiden Spitzen der Kegel sind, welche durch $K, K^{\prime}$ gelegt werden können; die erste führt $K$ in $K^{\prime}$, die zweite $K^{\prime}$ in $K$ über.

Man kann diese Trapsfornation ohne Weiteres anf die beliebigeq Formen:

$$
a_{x}=0, \quad \sum a_{i \alpha} x_{i} x_{k}=0,
$$

anwendev, welche einen Kegelschnitt $K$ bestimmen. Nimmt man die willkürliche Ebene:

$$
b_{x}=0
$$

hinzu, so sind die Spitzen der beiden kégtel, welche wir durch ihro Coordinaten:

$$
x_{1}{ }^{\prime}, x_{2}{ }^{\prime}, x_{3}{ }^{\prime}, x_{1}{ }^{\prime}, \quad x_{1}{ }^{\prime \prime}, x_{2}^{\prime \prime}, x_{3}^{\prime \prime}, x_{4}^{\prime \prime}
$$

bezeichnen, abhängig von den Wurzeln $\lambda_{1}, \lambda_{2}$ der quadratischep Gleichung:

$$
\Delta+2 \lambda\left(\begin{array}{l}
a \\
b
\end{array}\right)+\lambda^{2}\left(\begin{array}{l}
a b \\
a b
\end{array}\right)=0,
$$

in welcher $\Delta$ die Determinante der quadratischen Form, $\left(\begin{array}{l}a \\ b\end{array}\right),\left(\begin{array}{l}a b \\ a b\end{array}\right)$ die mit den $a_{i}, b_{i}$ bezüglich ein oder zweimal geänderten Determinanten sind, welche aus $\Delta$ entstehen. Ferner ist

$$
\begin{gathered}
\sum x_{i}^{\prime} x_{i}^{\prime} a_{i k}=2 \lambda_{i} a_{z^{\prime}} h_{z^{\prime}}, \quad \sum x_{i}^{\prime \prime} x_{k}^{\prime} a_{i k}-2 \lambda_{2} a_{*^{\prime \prime}} b_{x^{\prime \prime}}, \\
\sum x_{i}^{\prime \prime} x_{i k}^{\prime \prime} a_{i=1} .
\end{gathered}
$$

Unter Anwendung der Transformation (5) erhält man die folgende 
A. Voss. Ueber orthogonale Substitutionen.

Hermite'sche Transformation eines beliebigen Punktes ₹ des Kegelschnittes $K$ :

$$
\begin{aligned}
\eta_{i}=x_{i}^{\prime \prime} a_{x^{\prime}} b_{x^{\prime}}\left(a_{\xi} b_{x^{\prime \prime}}+b_{\xi} a_{x^{\prime \prime}}\right) & +x_{i}^{\prime} a_{x^{\prime \prime}} b_{x^{\prime \prime}}\left(a_{\xi} b_{x^{\prime}}+b_{\xi} a_{x^{\prime}}\right) \\
& -\xi_{i} a_{x^{\prime}}^{\prime} b_{x^{\prime}} a_{x^{\prime \prime}} b_{x^{\prime \prime}},
\end{aligned}
$$

aus welcher man leicht unter Voranssetzung von $a_{14}=a_{24}=a_{34}=0$, $a_{44}=1, a_{1}=0, a_{2}=0, a_{3}=0, a_{4}=1$ die Formeln herleitet, welche die Hermite'sche Transformation des Kegelschnittes $\sum a_{i k} x_{i} x_{k}=0$ geben.

Darmstadt, im December 1877. 